\title{
HOISAN 1.2: Programa informático para uso en Metodología Observacional
}

\author{
IT Program for Use in Methodology Observacional
}

\author{
HOISAN 1.2: Programa Informático para uso \\ em Metodología Observacional
}

\author{
Antonio Hernández Mendo*, José Antonio López López*, Julen Castellano Paulis**, Verónica Morales Sánchez*, José Luis \\ Pastrana Brincones*
}

*Universidad de Málaga y **Universidad del País Vasco (EHU-UPV)

\begin{abstract}
Resumen: La herramienta HOISAN (Herramienta de Observación de las Interacciones Sociales en Ambientes Naturales) es una aplicación informática que permite la codificación, registro, descripción y manipulación de grabaciones o del visionado en tiempo real desde una o varias cámaras. Permite trabajar con todos los tipos de datos: secuencias de eventos, de estados, mixtas, de intervalos de tiempo y de eventos multimodales. La métrica del registro observacional utiliza parámetros primarios y medidas derivadas o secundarias. Tiene la capacidad de analizar la producción verbal, así como de calcular distintos tipos de acuerdo e índices de correlación. El programa admite el intercambio de datos con programas específicos de uso en Metodología Observacional (SDIS-GSEQ, OBSERVER, THEME y MOTS), otros programas de carácter general (hojas de cálculo, paquetes estadísticos, procesadores de textos), y programas para el análisis cualitativo (Atlas.ti) y la exportación de los datos a PDF. En este trabajo se presentan, además, los resultados de un análisis de calidad del dato pertenecientes a una herramienta de observación para el fútbol 7.

Palabras claves: Observación, software, calidad del dato

Abstract: HOISAN tool (Tool Observation Social Interaction in Natural Environments) is a software application that enables the encoding, recording, description and handling of recordings or viewing in real time from one or more cameras. Can work with all data types: sequences of events, states, mixed, time intervals and multimodal events.

The observational record metrics and measures used parameters derived from primary or secondary.

It has the ability to analyze verbal output and calculate different types of
\end{abstract}

agreement and correlation indexes. The program supports data exchange with specific programs for use in observational methodology (SDIS-GSEQ, OBSERVER, THEME and MOTS), other general programs (spreadsheets, statistical packages, word processors), and programs for the analysis qualitative (Atlas.ti) and export of data to PDF.

In this paper we present also the results of quality analysis of the data belonging to an observation tool for football 7.

Key words: Observation, software, data quality

Resumo: A ferramenta HOISAN (Herramienta de Observación de las Interacciones Sociales en Ambientes Naturales) é uma aplicação informática que permite a codificação, registro e manipulação de gravaçôes o filmagens em tempo real, desde uma o várias câmaras de vídeo. Esse sistema permite trabalhar com todos os tipos de dados: seqüências de eventos, de estados, mistas, de intervalos de tempo e de eventos multimodais. A métrica deo registro observacional utiliza parâmetros primários e medidas derivadas o secundárias. Têm a capacidade de analisar a produçáo verbal, assim como de calcular distintos tipos de acordo e índices de correlação. O programa admite o intercambio de dados de acordo com programas específicos de uso em Metodologia Observacional (SDIS-GSEQ, OBSERVER, THEME y MOTS), outros programas de caráter geral (páginas de calculo, packs estatísticos, processadores de textos), programas para a análises quantitativa (Atlas.ti) e a exportação dos dados para PDF. Nesse trabalho apresentamos os resultados de uma análise de qualidade dos dados de uma ferramenta de observação para o futebol sete.

Palavras chave: Observação, software, qualidade do dado.

\section{Introducción}

La Metodología Observacional (MO) ha evolucionado notablemente en las últimas décadas (Anguera, Blanco, Losada y Hernández Mendo, 2000; Anguera, Blanco Villaseñor, Hernández Mendo, y Losada, 2011), esta perspectiva queda maximizada en el aspecto tecnológico, donde la proliferación de software (Hernández Mendo, Anguera y Bermúdez-Rivera, 2000; Castellano, Perea, Alday y Hernández Mendo, 2008) y tecnologías de diversa índole han aumentado notablemente (Castellano, Perea y Hernández Mendo, 2011). Aunque si bien es verdad que se siguen utilizando herramientas que no se adaptan a los diversos contextos sociales, acarreando los

Dirección de contacto: mendo@uma.es consiguientes problemas de validez, que provocan una considerable pérdida en las garantías científicas.

Ninguna de las aplicaciones que han sido analizadas previamente (Castellano, Perea y Hernández-Mendo, 2008) aglutinan todas las ventajas que proporcionan el conjunto de ellas en lo referido a la configuración de herramientas de codificación, visionado, registro y análisis o salida de los datos, haciendo necesaria la utilización de diferentes aplicaciones para cada una de las etapas de investigación: observación, registro, calidad del dato, análisis y elaboración de informes.

En relación a la configuración de la herramienta de observación diseña ad hoc por el investigador, una de las lagunas que algunas aplicaciones informáticas presentan es el carácter 
cerrado de las mismas (Castellano, Perea, Alday y Hernández Mendo, 2008), es decir, cuando la propia aplicación impone los códigos o un número limitado (p.e. Nacsport, http:// nacsport.com, en sus versiones más básicas) que se deben utilizar para llevar a cabo el registro. Además las aplicaciones no siempre permiten seguir de forma nítida, clara y sencilla el proceso de estructuración categorial (definición de núcleos categoriales, grados de apertura y participantes) así como las pautas previas de los diseños observacionales (Anguera, Blanco Villaseñor, Hernández Mendo, y Losada, 2011), siendo este uno de los requisitos a los que debería responder un software en este ámbito. Además, los programas de observación actuales utilizan sistemas de categorías más o menos ortodoxos, algunos permiten la inclusión de modificadores (Observer XT, http://www.noldus.com/human-behavior-research/products/2/ the-observer- $x t$ ), pero alejados en la mayoría de los casos de la casuística de los tipos de datos existentes en MO (Bakeman y Quera, 1995), como son: secuencias de eventos, secuencias de estados, secuencias mixtas, secuencias de intervalo y secuencias de eventos multimodales. En ocasiones, además, el registro de los datos no mantiene la cronología de los acontecimientos (Castellano et al, 2008), lo que imposibilita posteriores análisis del tipo secuencial o de coordenadas polares. Por toda esta casuística, en relación al andamiaje conductual, gran parte de las herramientas dejan espacios sin cubrir.

La ventaja que supuso la digitalización del video abrió paso a la posibilidad de implementar interface de video en las aplicaciones informáticas, cuestión que no se contemplaba con anterioridad (p.e. Transcriptor de Hernández Mendo, Ramos, Peralbo y Risso,1993 o Codex de Hernández Mendo, Anguera y Bermúdez-Rivera, 2000). El rápido avance en el desarrollo de las tecnologías informáticas y del tratamiento de las imágenes, que hacen de estos últimos diez años la década tecnológica, no están recogidos ni implementados en la mayor parte del software existente. Así, desde los programas más comerciales, como Observer XT, hasta los programas más aplicados al ámbito deportivo como el Softory match ( $h t t p: / /$ softory-match.software.informer.com), LongoMatch (bttp:// longomatch.org), PosiCAP (http://posicap.software.informer. com/2.1), FOCUS (http://www.elitesportsanalysis.com), ELAN (http://www.lat-mpi.eu/toolslelan), Interplay Sport, Sportstec (http://www.sportstec.com/Products_Sportscode), Sports Analytica (http://www.briggspalmer.com/new/index.html), Match Analysis (http://matchanalysis.com), Dart Fish (http:// www.dartfish.com), Match Vision Studio (www.observesport. com) por mostrar algunos ejemplos, utilizan un solo video desde el cual capturar la observación. La implementación de la observación de varios vídeos al estilo de Kinovea (bttp:// www.kinovea.org/en) del mismo evento realizado desde perspectivas diferentes mejoraría sin duda las opciones de enriquecer y mejorar la calidad de las investigaciones.

En tercer lugar, respecto al tratamiento de los datos re- cogidos desde contextos naturales, las necesidades del investigador en este ámbito pasan por disponer de un inmediato feed-back sobre una cuestión de máxima relevancia en $\mathrm{MO}$ como es la constatación de la fiabilidad, validez y precisión de herramientas y de observadores. Los programas que existen en la actualidad permiten observar el flujo comportamental, pero no disponen en la mayoría de las ocasiones la opción de exportarlos directamente al formato que se pide en los programas estadísticos (EduG,TG, SAS, SPSS, Atlas.Ti, Theme o $S D I S-G S Q)$ para realizar los análisis correspondientes. Además, solo unos pocos (Observer XT, SDIS-GSEQ) permite calcular de forma directa el índice Kappa de Cohen, y otros índices para estimar la calidad del dato registrado. Además en ninguno de las aplicaciones revisadas la exportación de los datos

Por otro lado, el ámbito de aplicación donde la observación se convierte en imprescindible es el ámbito del deporte profesional, observar y analizar a los equipos rivales y el juego del propio equipo es cada vez más demandado por técnicos y entrenadores. El software existente está orientado a la investigación (Observer, MOTS) o bien al ámbito del deporte profesional (Interplay Sport, Sportstec (SportsCode), The Observer Video-Pro, Sports Analytica, Match Analysis, Nac Sport, Dart Fish, Match Vision Studio y Softory Match). Sin embargo se desconoce la existencia de aplicaciones que satisfagan la vertiente más profesional al tiempo que la investigadora en su implementación, lo que significaría el contrapunto donde unir investigación y práctica.

Finalmente, en el análisis de estos desequilibrios es donde se sitúa el origen de este software, intentando dar respuesta a una parte importante de las necesidades de investigación y de aplicación en el ámbito más deportivo, aportando la versatilidad y practicidad al tiempo que riguroso y sistemático en todos los procesos que permite realizar.

\section{Descripción del programa HOISAN y sus opciones}

Características técnicas y opciones de menú. Las principales características técnicas de este programa que se presenta son:

- Herramienta implementada en .NET y usando el lenguaje C\#.

- Trabaja con los principales formatos de vídeo

- Puede usar sistema PAL o NTSC en el visionado de los vídeos.

- Permite la codificación, registro, descripción, manipulación de grabaciones o del visionado en tiempo real desde una o varias cámaras. Cuando se realiza el registro, se almacena automáticamente el tiempo, tanto en segundos como en frames, del lugar del vídeo donde se ha producido.

- Los datos pueden ser importados desde otra base de da- 
tos Observacional propia y desde el MOTS (Castellano, Perea, Alday, y Hernández Mendo, 2008).

- La herramienta está traducida a varios idiomas (Español, Inglés, Portugués y Francés).

- Se pueden seleccionar las columnas a imprimir.

- Posee notificador de avisos programable con ventanas emergentes.

- Posee un capturador y un editor de frames, pudiendo utilizar también vídeos para poder editarlos dibujando (líneas, círculos, elipses, rectángulos, texto) y seleccionando regiones y diferentes colores. La imagen resultante se podrá exportar a diferentes formatos de imagen.

- Elaboración de gráficas relativas a los distintos análisis realizados.

- Análisis de la producción recogida en el campo observaciones (principalmente producción verbal o comentarios del observador).

Después de instalar la aplicación y ejecutarla, en la parte superior aparecerá el menú principal con las distintas opciones (figura 1):

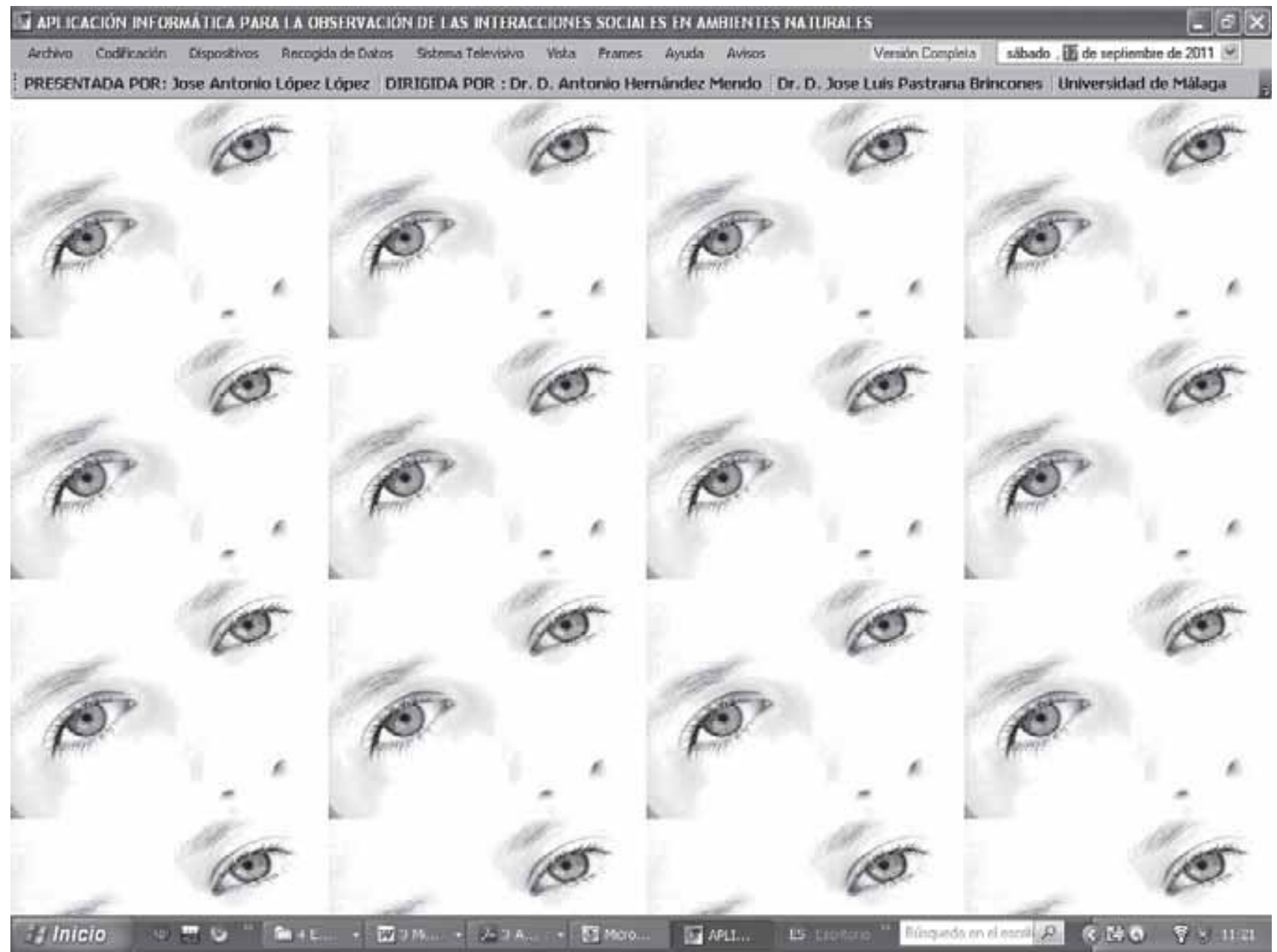

Figura 1: Pantalla principal del programa HOISAN

A continuación se presentan las opciones del menú principal. Cada opción aparece recogida en una tabla con la imagen y una breve descripción. 
Tabla 1: Opciones del Menú Archivo

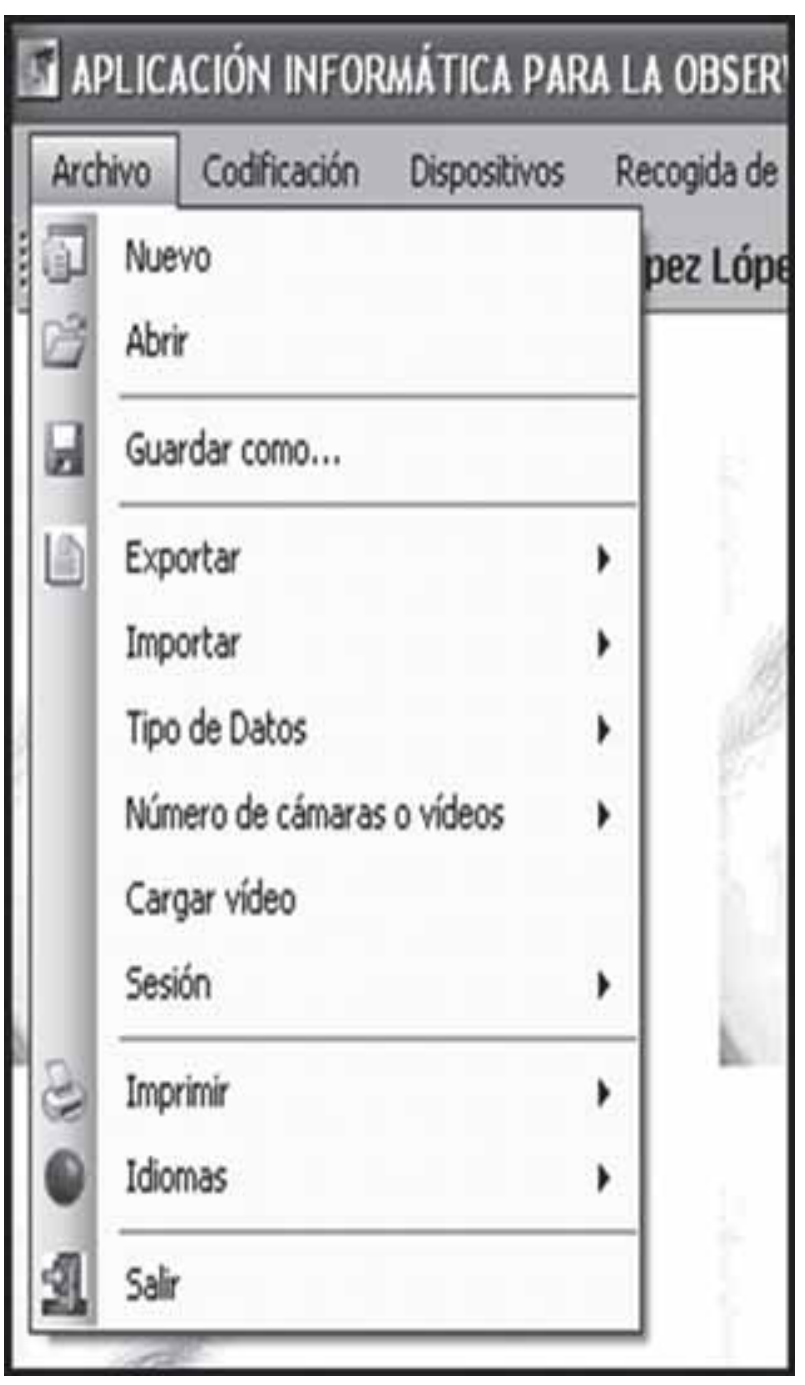

Figura 2: Opción Archivo

Tabla 2: Opciones de los Menús Codificación y Dispositivos

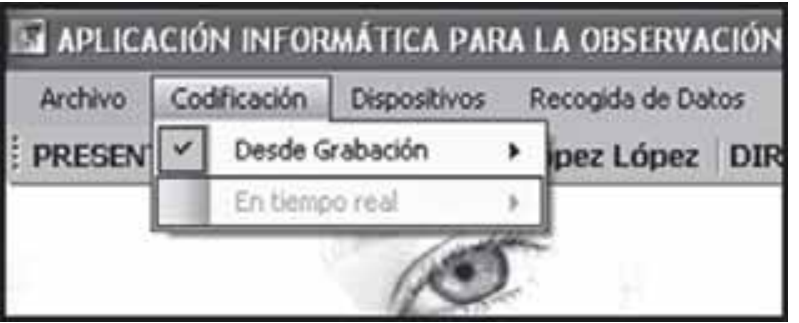

Figura 3: Opción Codificación
Nuevo: Creación de una nueva base de datos Observacional.

Abrir: Abrir una base de datos Observacional ya creada.

Guardar como: Guardar una base de datos Observacional abierta, con otro nombre.

Exportar: Exportar los datos a distintos programas como son (PDF, Excel, Word, HTML, CSV, SDIS-GSEQ. Observer y Theme.

Importar: Importar desde otra base de datos de formato propio. Tipos de Datos: Los datos con los que trabaja el programa (secuencias de estados, eventos, mixtas, intervalos de tiempo y eventos multimodales).

Número de cámaras o vídeos: Elección del número de cámaras o vídeos a analizar al mismo tiempo.

Cargar vídeo: Opción para cargar los distintos vídeos a observar. Sesión: Selección de la sesión a observar.

Imprimir: Impresión de los distintos datos almacenados.

Idiomas: Elección de los distintos idiomas en los que está traducido el programa (Español, Inglés, Portugués y Francés).

Salir: Salir del programa.
Desde Grabación: Control de los vídeos grabados y almacenados en el sistema (parar, pausar, iniciar).

En tiempo real: Control de las cámaras usadas para observar en tiempo real. Se habilitaría el menú Dispositivos.

Dispositivos de Vídeo: Configuración de la imagen de las cámaras. Dispositivos de Audio: Configuración del audio de las cámaras.

Previsualizar: Ver la imagen de las cámaras.

Grabar: Empezar la grabación de las cámaras.

Parar Grabaciones: Parar la grabación de las cámaras. 


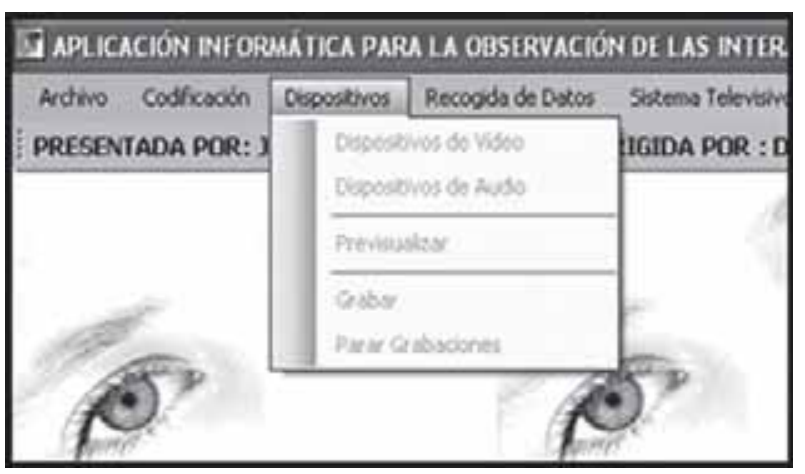

Figura 4: Opción Dispositivos

Tabla 3: Opción Recogida de Datos

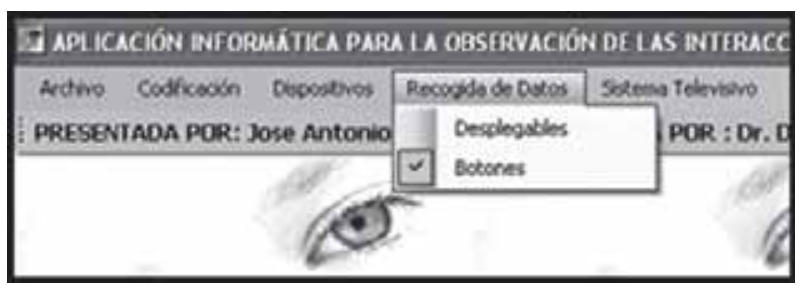

Figura 5: Recogida de Datos

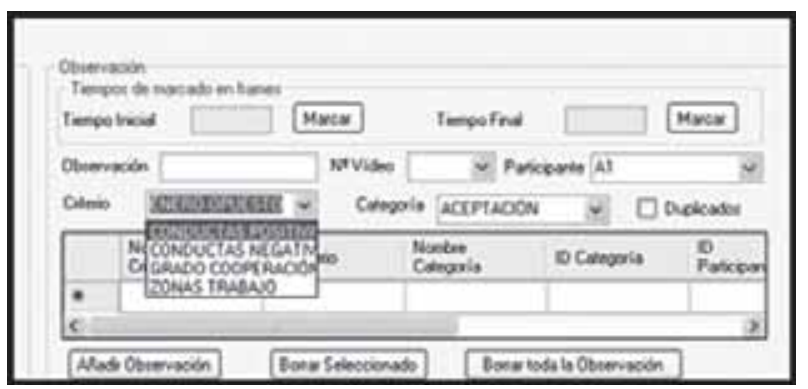

Desplegables: Para la selección de los distintos códigos en la observación se utiliza desplegables.

\begin{tabular}{|c|c|c|c|c|}
\hline \multicolumn{5}{|c|}{ CONDUCTAS POSITIVAS GENERO } \\
\hline ACEPT & RELAC & COLAB & AYUD & SINC \\
\hline \multicolumn{5}{|c|}{ CONDUCTAS NEGATINAS GENERO } \\
\hline SEPA & $\mathrm{AECH}$ & VERB & FISICA & SINC \\
\hline \multicolumn{5}{|c|}{ GRADO COOPERACION ACIMDAD } \\
\hline COOP & TRAN & ESQUI & NO & SINC \\
\hline \multicolumn{4}{|c|}{ ZONAS TRABANO } & \\
\hline PAINC & INTER & MARGI & SINC & \\
\hline
\end{tabular}

Botones: Para la selección de los distintos códigos en la observación se emplean botones.

PAL: Configuración cada segundo con 25 frames. NTSC: Configuración cada segundo con 30 frames.

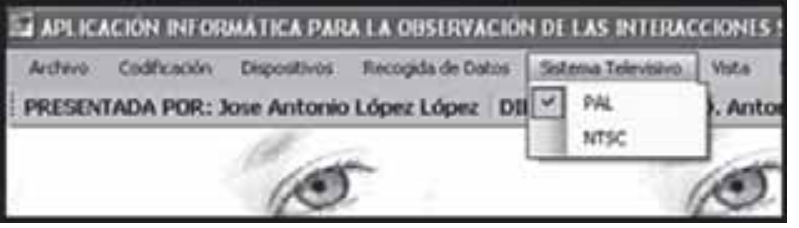

Figura 6: Sistema Televisivo 
Tabla 5: Opción Vistas

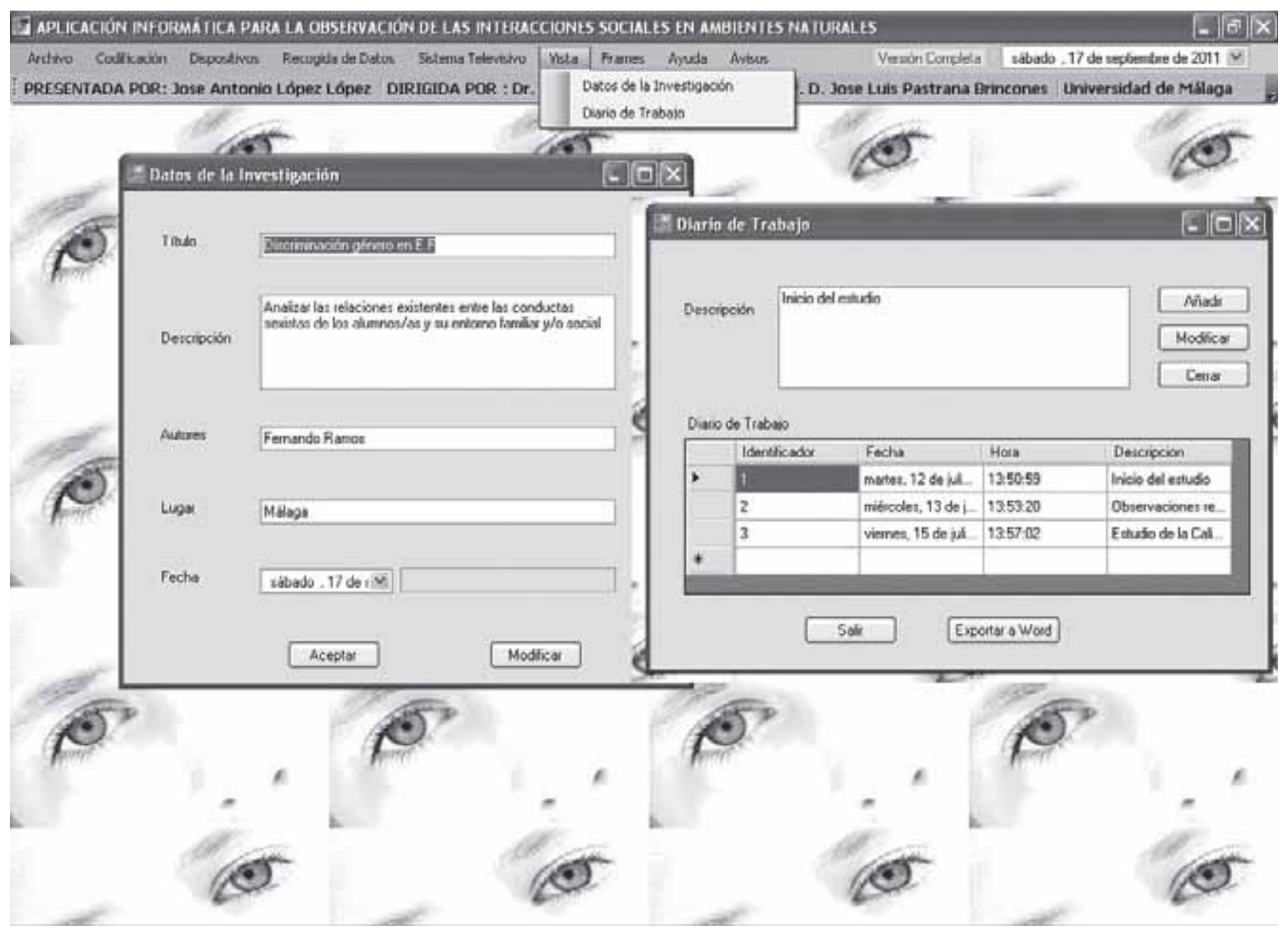

Figura 7: Datos de la Investigación y Diario de Trabajo

Datos de la Investigación: Almacena los principales datos de la investigación a desarrollar con posibilidad de modificar

Diario de Trabajo: En esta ventana se almacena el trabajo realizado diariamente, por cada registro se almacenará automáticamente la fecha y la hora de almacenamiento. 
Tabla 6: Opción Frames

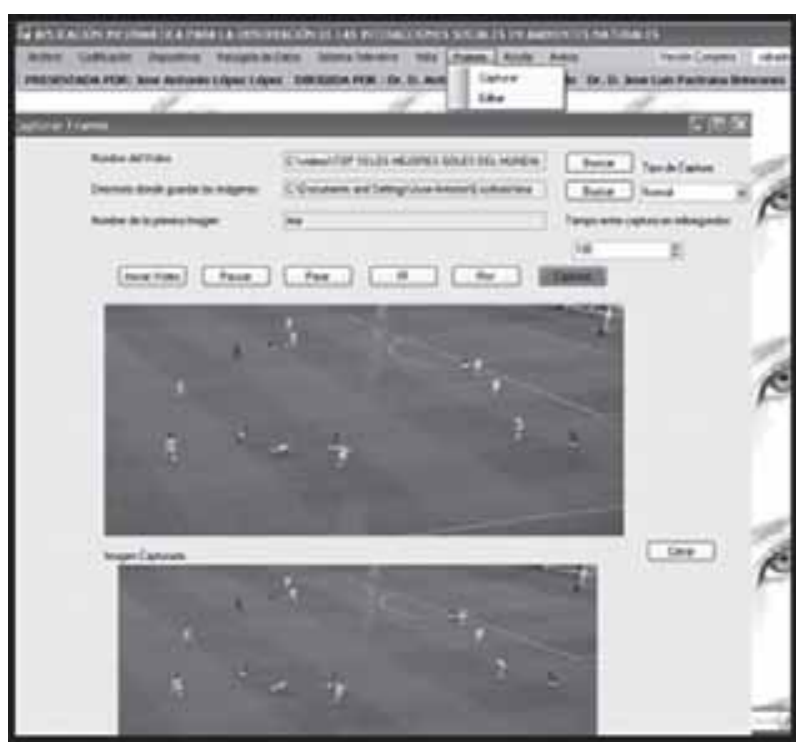

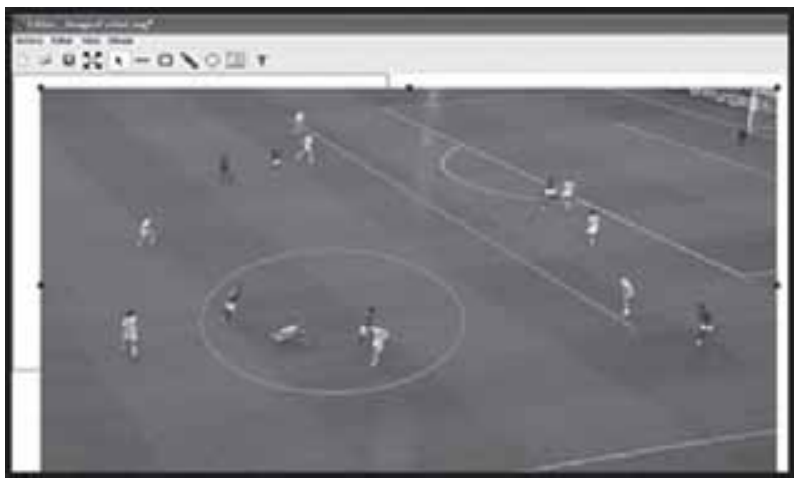

Figura 9: Editor de Frames

Figura 8: Capturador de Frames

Capturar: En el lado izquierdo, está situado el menú para capturar los distintos frames de un video observado, almacenando el frame en formato imagen para su posterior edición.

Editor: En el lado derecho de la imagen, se puede editar la imagen capturada con el editor de frame.

Tabla 7: Opción Ayuda

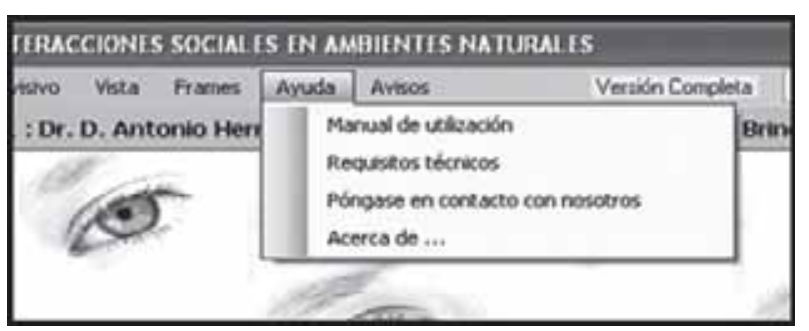

Figura 10: Opción Ayuda
Manual de utilización: Manual en formato PDF para la utilización Requisitos técnicos:

Póngase en contacto con nosotros: Información para comunicarse con los creadores de la aplicación.

Acerca de...: Información de la aplicación. 
Tabla 8: Opción Avisos

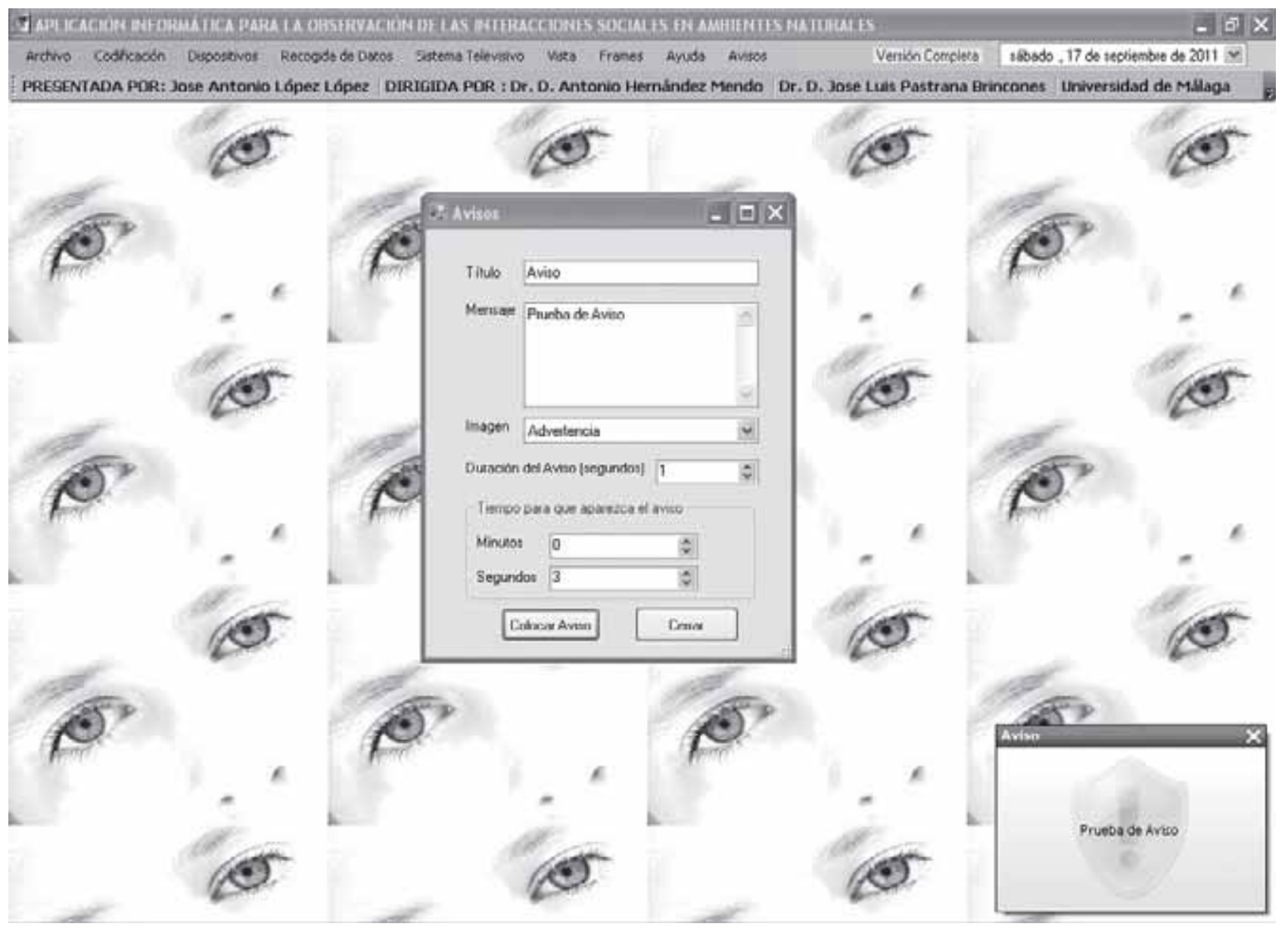

Figura 11: Opción Avisos

Avisos: Opción del menú donde se puede programar la colocación de avisos rellenando las opciones: Título, Mensaje, Imagen, Duración del aviso y Tiempo para que aparezca el aviso. Aparecerá una ventana emergente con el aviso, trascurrido el tiempo de espera configurado, en la parte inferior derecha de la pantalla.

Tras describir las distintas opciones del menú principal, y tras cargar una base de datos ya creada, aparecerá una pantalla de Recuperación de la última Sesión guardada. En la figura 12 aparecerá cada una de las opciones de la observación guardada como son: Tipo de dato, Fecha y Hora de la Ob- servación; así como los Vídeos y la posición donde se detiene la observación. Cuando se cierra el programa, preguntará si guarda los vídeos observados en su última posición. 
Figura 12: Recuperación de Sesión

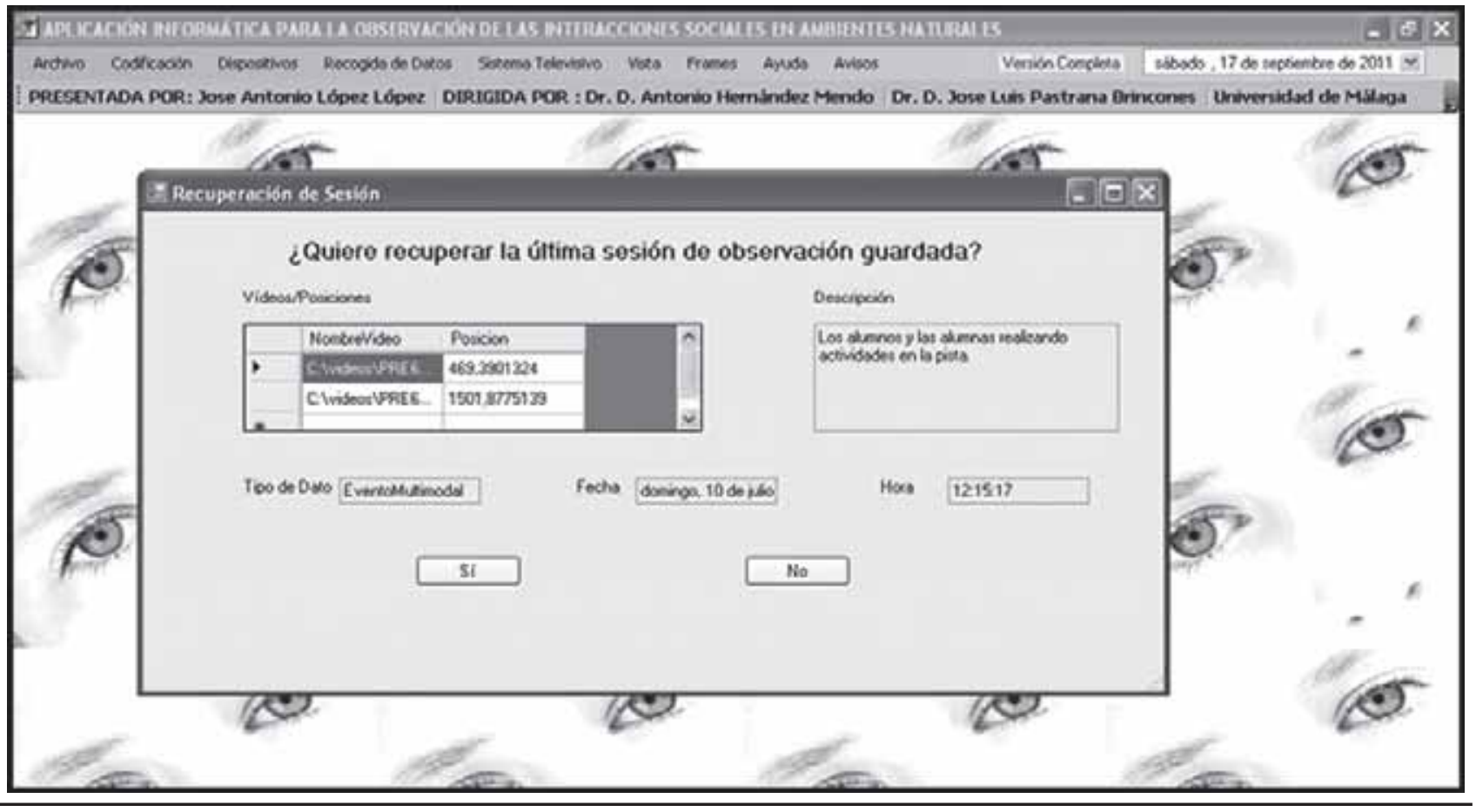

Después se debe seleccionar el tipo de dato que se utilizará pinchando en el menú (ArchivoàTipos de DatosàSelección del adecuado). Para cada tipo de dato aparecen cuatro pesta- ñas (Tipo de dato, Observaciones, Análisis o Descripciones y Búsquedas). Cada pestańa corresponde a una tarea que se puede realizar con el tipo de dato. Ver tabla 9.

Tabla 9: Tipos de Datos: Secuencias (Eventos, Estado, Mixtas y de Intervalos)

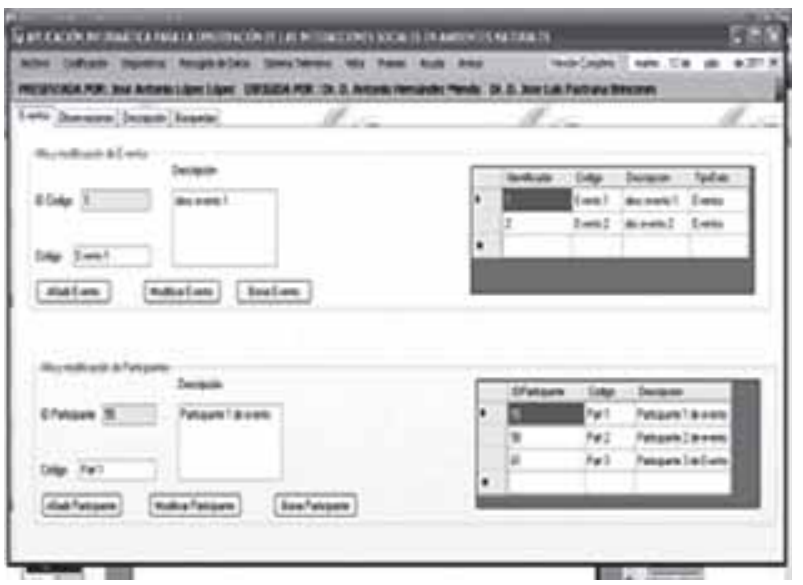

Figura 13: Secuencias de Eventos

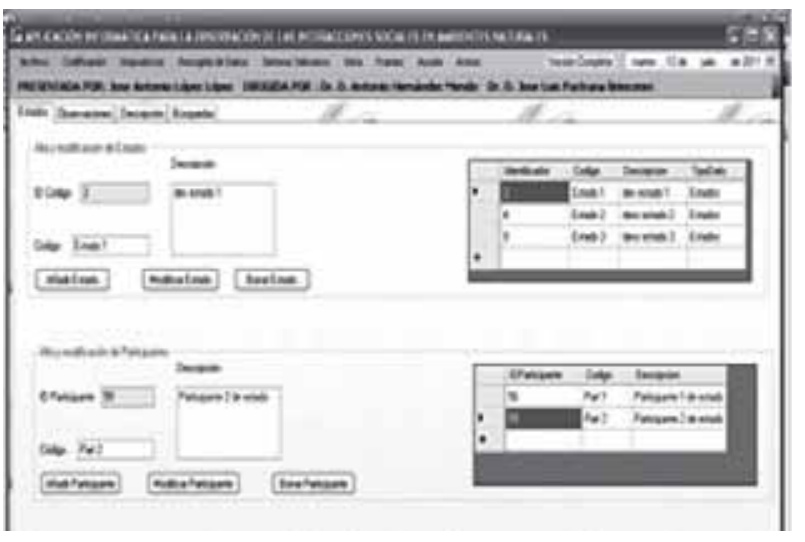

Figura 14: Secuencias de Estado 


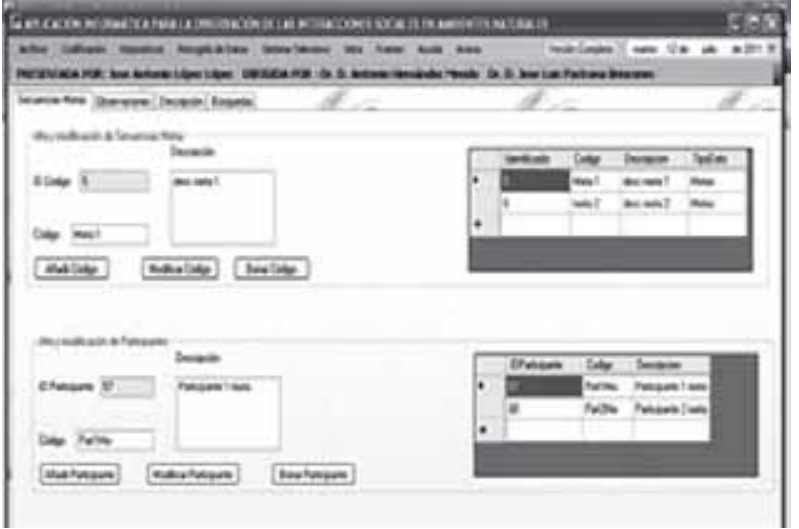

Figura 15: Secuencias Mixtas

Cada tipo de dato presenta las mismas características. A modo de ejemplo, se utiliza el tipo de datos "Eventos Multimodales". En la Figura 17, se recoge la primera pestańa que corresponde a la entrada de los distintos códigos. Se puede dividir en distintas partes:

- Alta y modificación de criterios: Para añadir, modificar y borrar cada uno de los criterios que contendrá la investigación.

- Alta y modificación de Participantes: Para añadir, modificar y borrar los distintos participantes usados en el

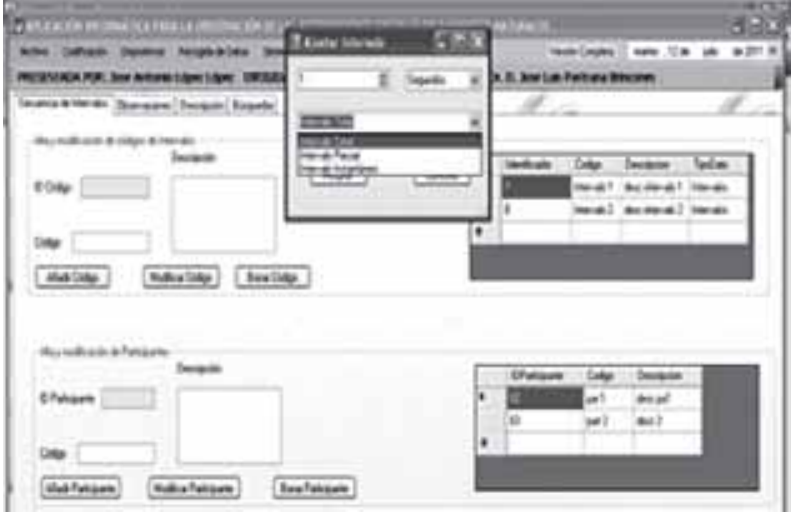

Figura 16: Secuencias de Intervalos

estudio.

- Alta y modificación de categorías: Tras crear un criterio, en esta parte podrán ańadir las distintas categorías que poseerá el criterio seleccionado.

- Alta y modificación de Grados de Apertura: Tras crear una categoría, se podrá asignar distintos grados de apertura.

- Ver todos los criterios con sus categorías: Se puede visualizar todos los criterios con sus distintas categorías.

Figura 17: Eventos Multimodales

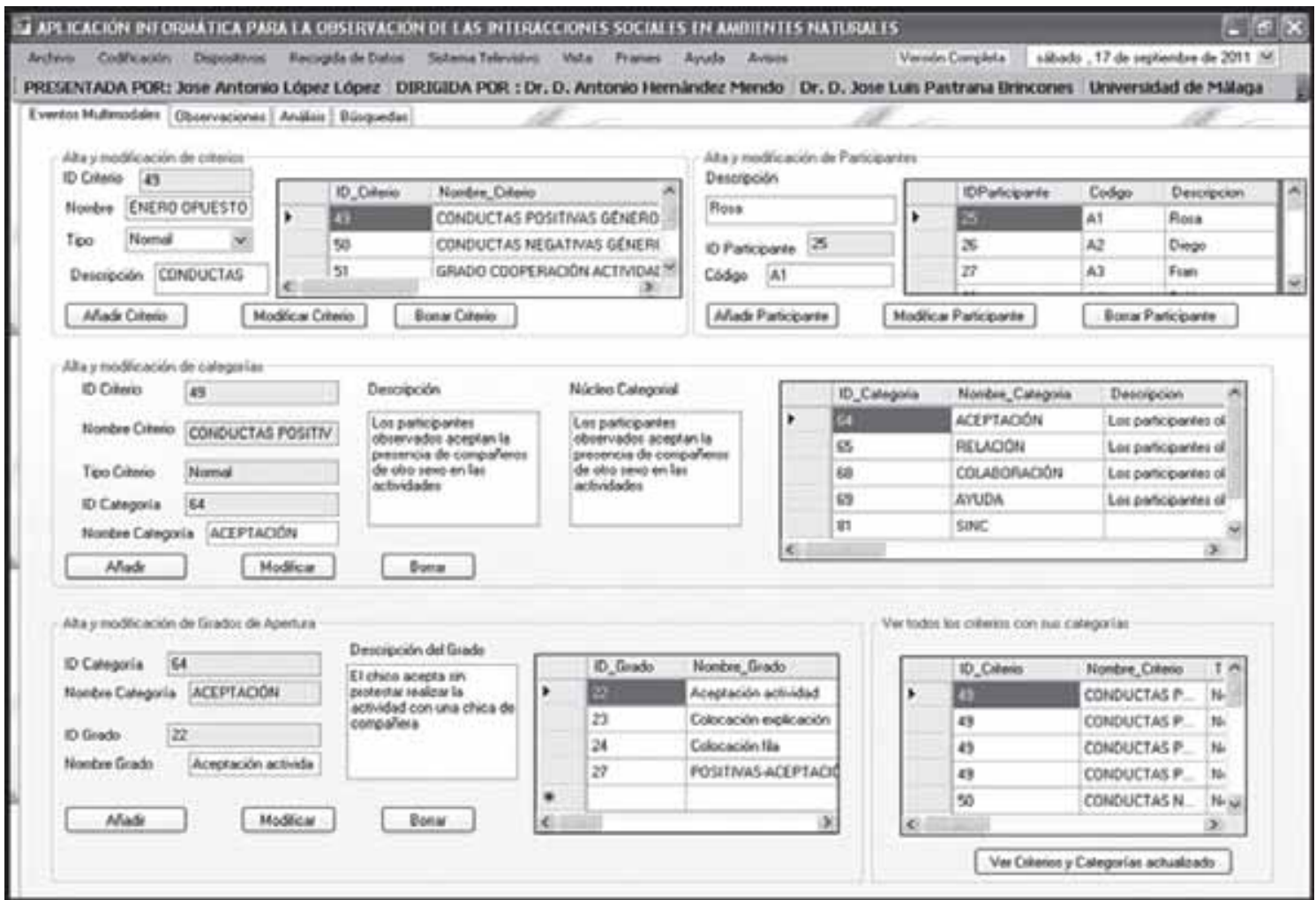


En la siguiente pestaña, Figura 18, aparecen las "Observaciones", es la pantalla donde se deben registrar las observaciones que se pretenden realizar. Para registrar una observación se deben seguir este proceso:

Se debe seleccionar el vídeo o los vídeos de donde se desea observar.

Marcar el tiempo Inicial y el Tiempo Final de la conducta.

Rellenar, de forma optativa, el campo Observación con alguna descripción.

Selección el participante que se desea observar.

Se selecciona el Criterio con su correspondiente Categoría a observar.

Todo el proceso anterior se guardaría en la tabla situada abajo a la derecha de la Figura 17, donde se pueden añadir más filas de la misma manera.

Finalmente pinchando en el botón "Añadir Observación" se almacena el registro.

Las observaciones registradas, pueden ser comprobadas en la tabla inferior izquierda de la figura 18. Cuando se pincha sobre la observación, se puede realizar una modificación sobre los tiempos de registro.

Figura 18: Observaciones

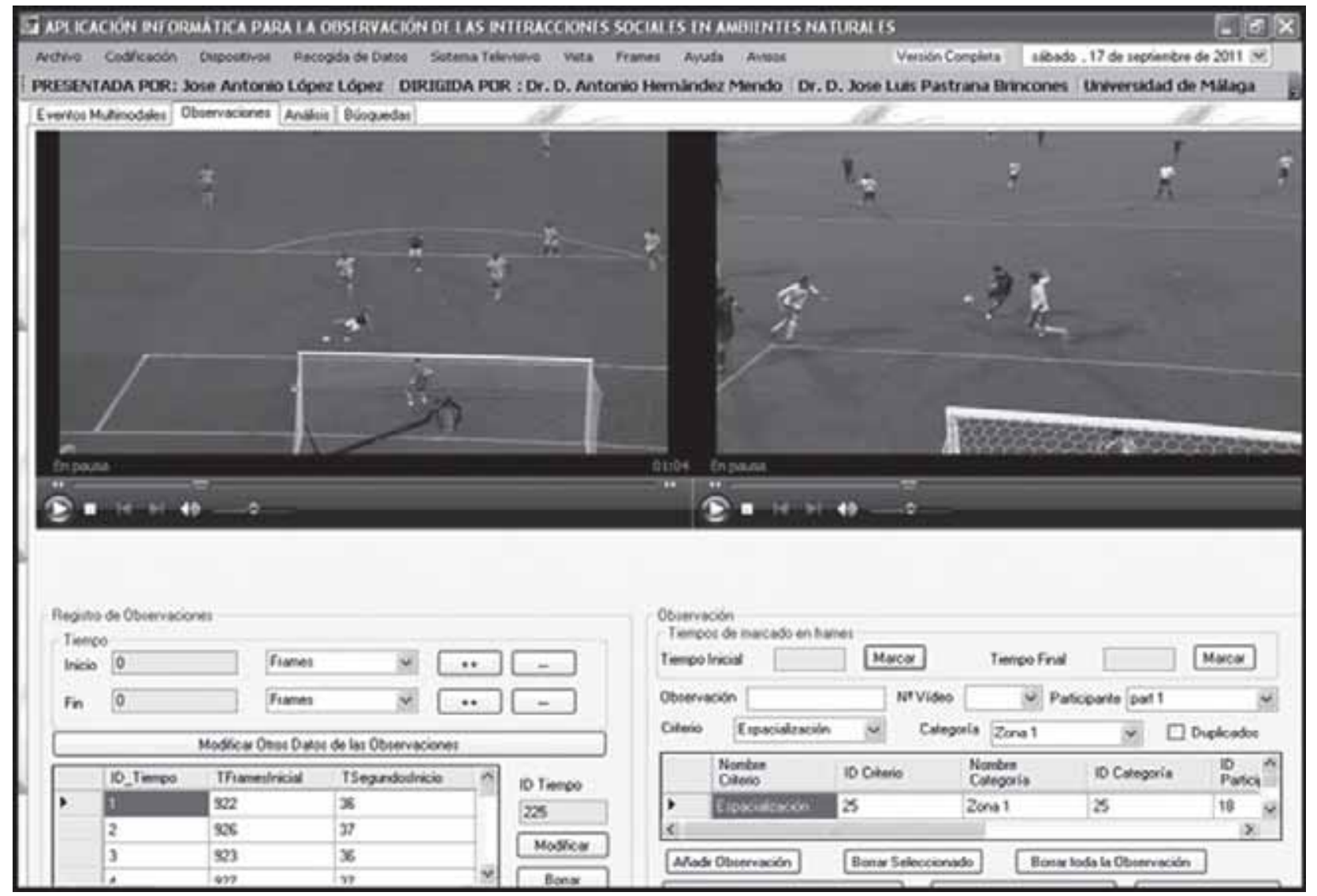

Si se desea modificar más datos dentro de la observación, se puede realizar pinchando en el botón "Modificar Otros Datos de las Observaciones". Ver figura 19. 
Figura 19: Modificar Observaciones

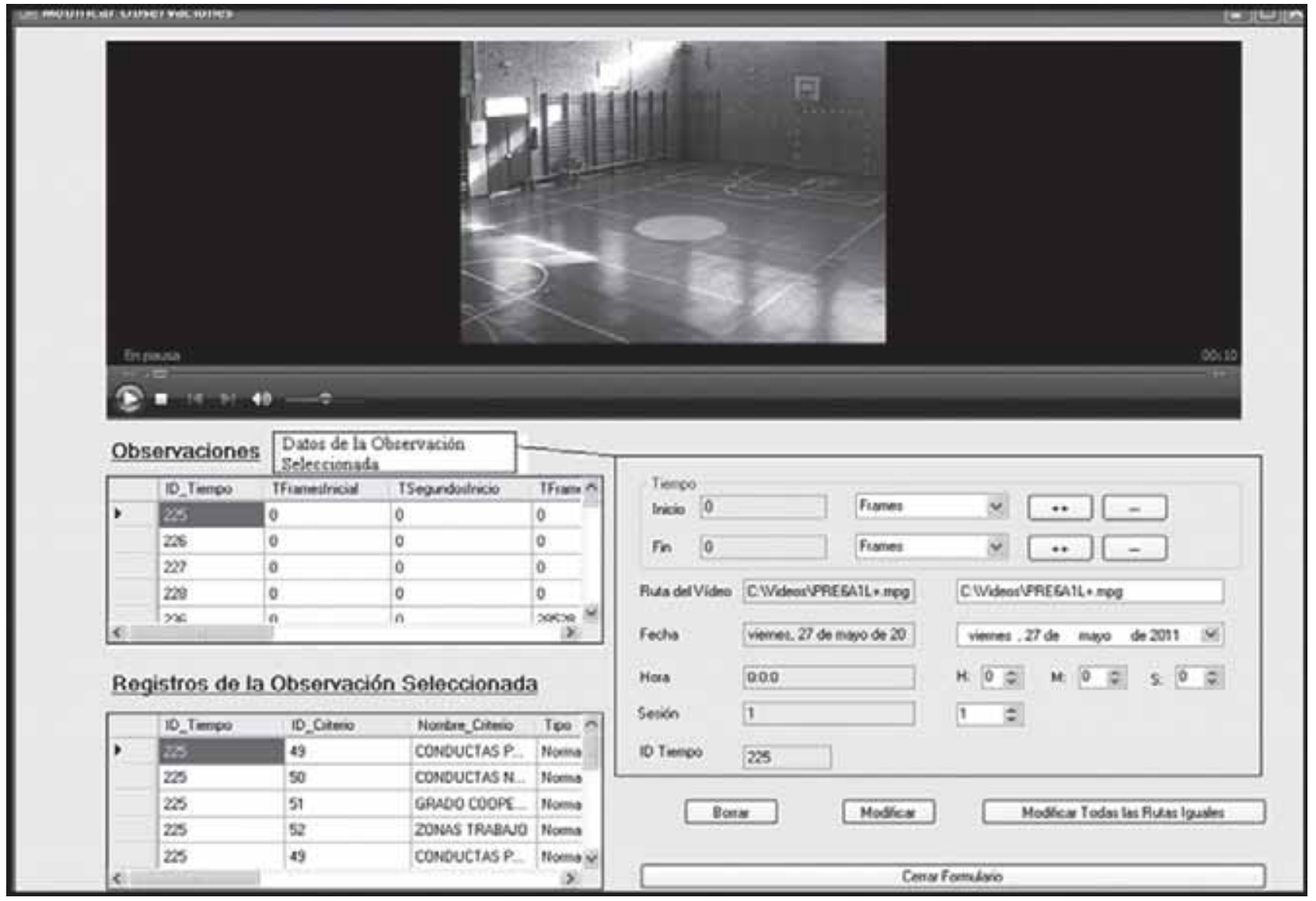

En la pestaña de "Análisis" o "Descripción”, (ver Figura 20), se realiza el cálculo de parámetros primarios (frecuencias, lapsos y latencias) y secundarios (tasas, frecuencias relativas y duraciones).

En la parte superior derecha de la Figura 20, en el grupo de opciones "Observaciones" se puede realizar un primer análisis de los registros que figuren en ese campo, generalmente la producción verbal. Opciones como "Contar palabras", "Contar letras", "Estadística”, etc.... También se puede exportar los datos a Excel y al programa Atlas.ti 6.2. Véase la Figura 21.

En la parte inferior de esta pantalla (Figura 20), se realiza el análisis de calidad del dato a través del cálculo del coeficiente de Pearson, los Coeficientes de Kappa de Cohen y Pi y el cálculo de diversos coeficientes de acuerdo (Global Total, Aleatoria y Neta). 
Figura 20: Análisis

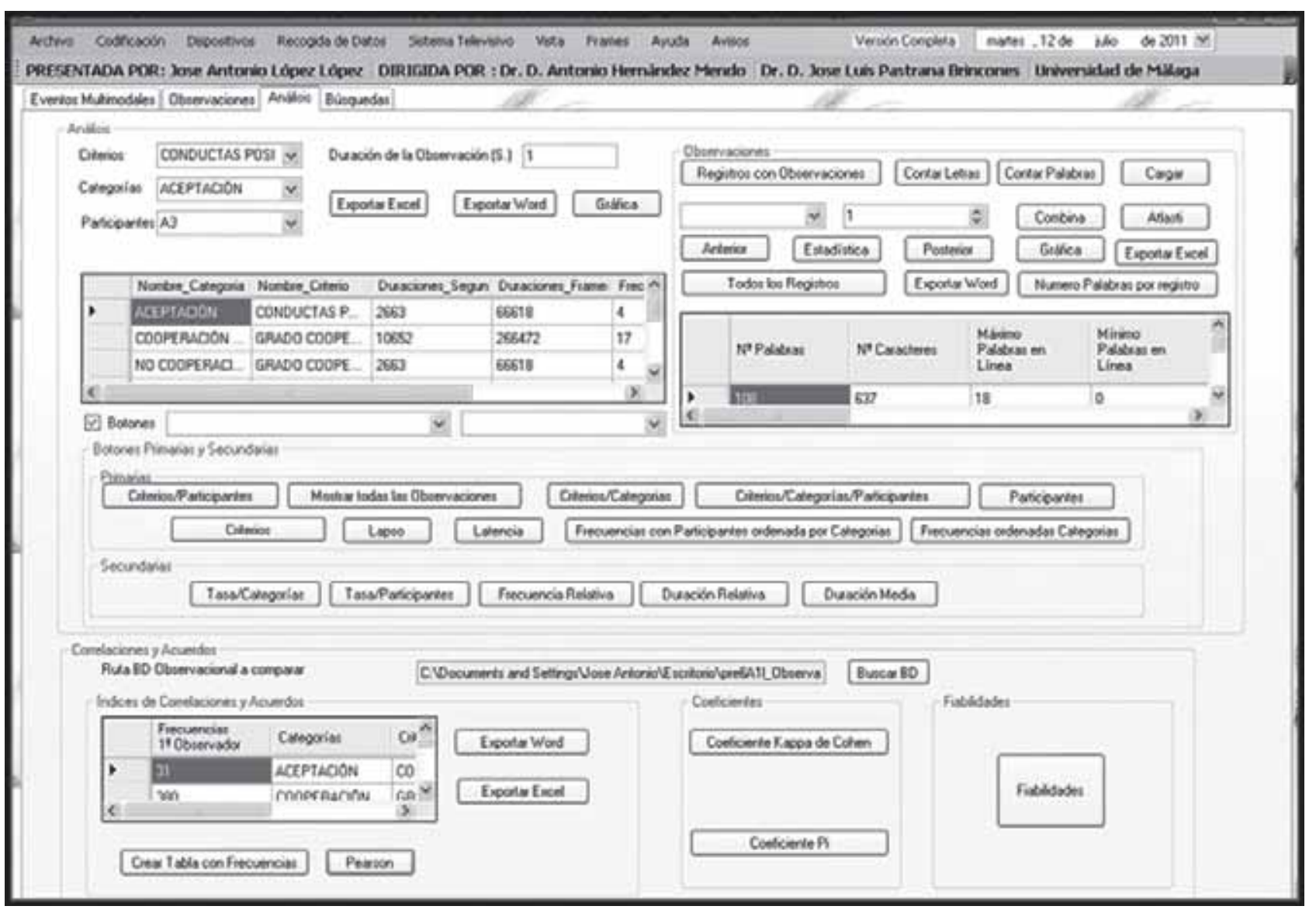


Figura 21: Exportación de la Producción Verbal a Atlas.ti 6.2 y a Excel

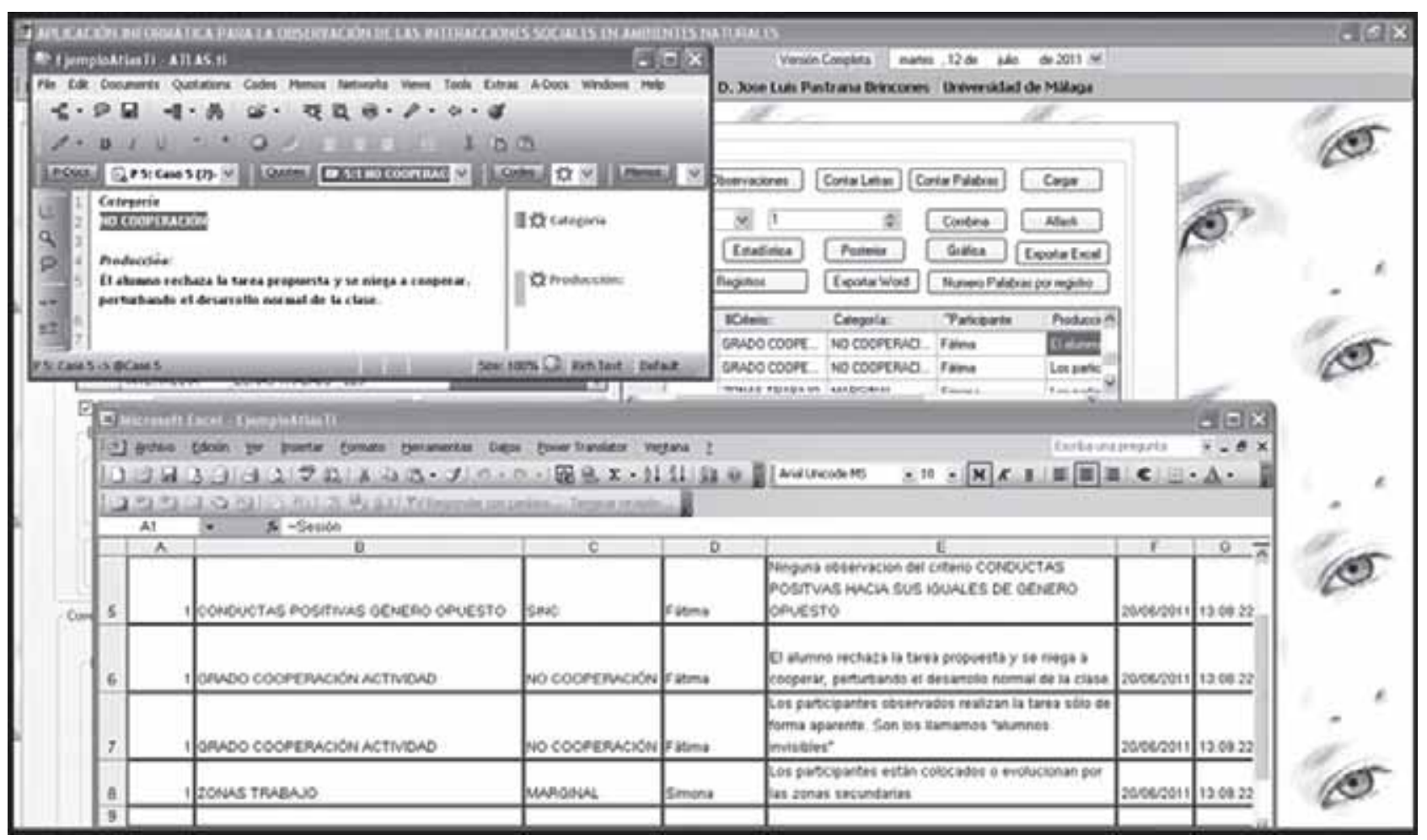

Para el cálculo del Coeficiente de Kappa se puede optar por calcular tres tipos de Coeficientes. Véase la siguiente Tabla 10.

Tabla 10: Cálculo Coeficiente Kappa de Cohen

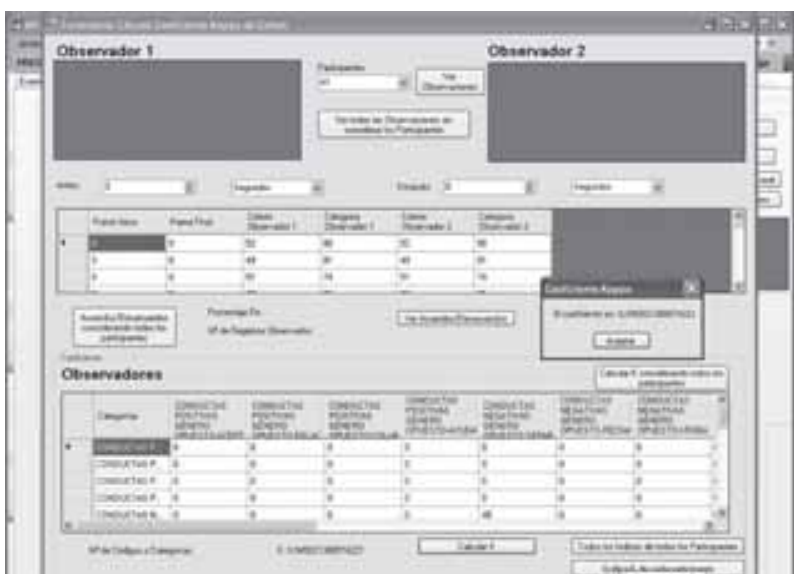

Figura 22: Cálculo Coeficiente Kappa de Cohen
Coeficiente de Kappa considerando todos los participantes. Coeficiente de Kappa sin considerar los distintos participantes. Cálculo de un Coeficiente de Kappa por cada participante. 
Tabla 11: Cálculo del Coeficiente de Pi y de Fiabilidades

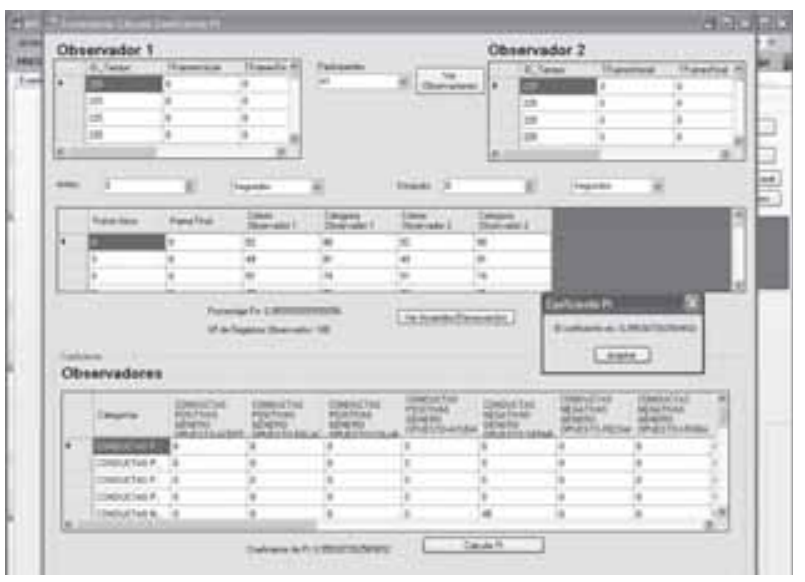

Figura 23: Coeficiente de Pi

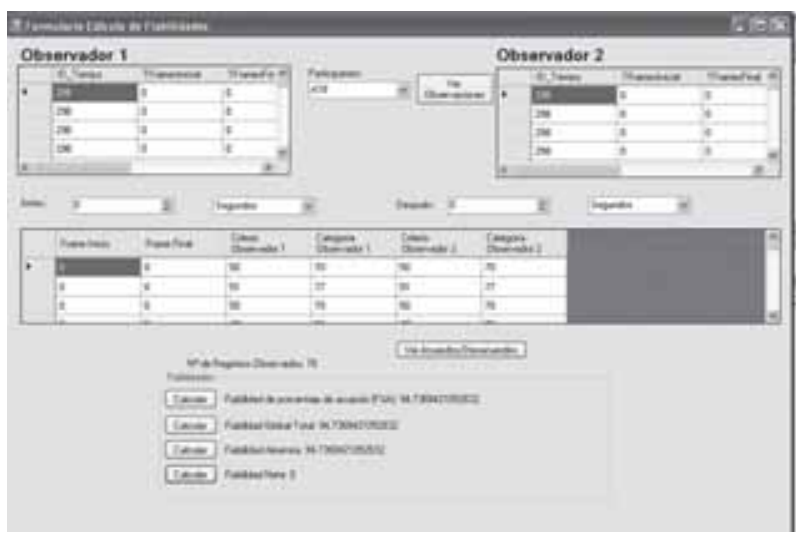

Figura 24: Fiabilidades

En la imagen izquierda se puede observar el cálculo del Coeficiente de Pi. En la imagen de la derecha se recoge el cálculo de Fiabilidades (Porcentaje de Acuerdo, Global Total, Aleatoria y Neta).

La aplicación posee la capacidad de crear distintas gráficas de los análisis desarrollados. Como ejemplo, se muestra la Tabla 12 con las Figuras 25 y 26, dos de los análisis con sus correspondientes gráficas generadas. Dichas gráficas pueden guardarse como imagen o directamente imprimirse.

Tabla 12: Gráficas del Cálculo de Frecuencias de Categorías y de Palabras

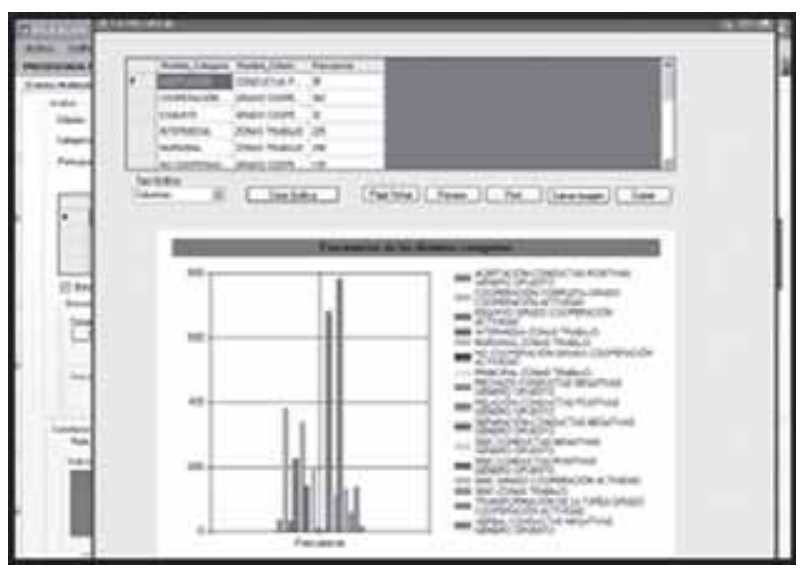

Figura 25: Frecuencias de Categorías

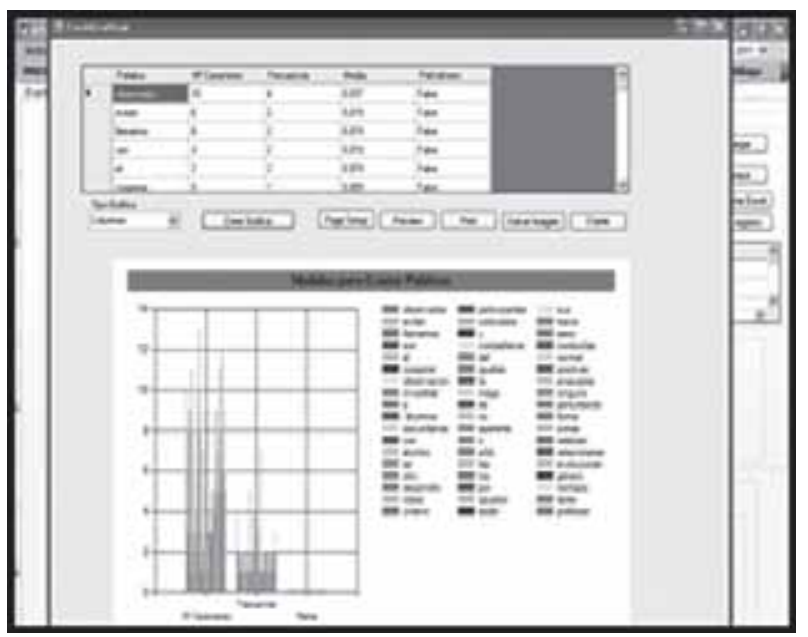

Figura 26: Frecuencia de Palabras

La Figura 25 muestra la gráfica de las frecuencias de las distintas categorías del estudio. La Figura 26 muestra la frecuencia de las palabras de la producción Verbal del estudio.

En la pestaña "Búsquedas", (ver Figura 27), aparecen las funciones de búsqueda de los registros almacenados que cumplen con unos criterios que sirven de filtro. En la parte inferior derecha se almacenará los n-criterios que se van empleando en la búsqueda. Tras realizar la búsqueda en la parte superior derecha aparece en la tabla los registros que cumplen con la búsqueda. Al pinchar en el registro aparecerá el vídeo con el tiempo de duración de la conducta y en la tabla inferior derecha cada uno de los registros asignados a la observación. Debajo de la tabla superior derecha, aparece una sección llamada Tiempos que utiliza para modificar el tiempo del registro almacenado para su visionado. 
Figura 27: Búsquedas

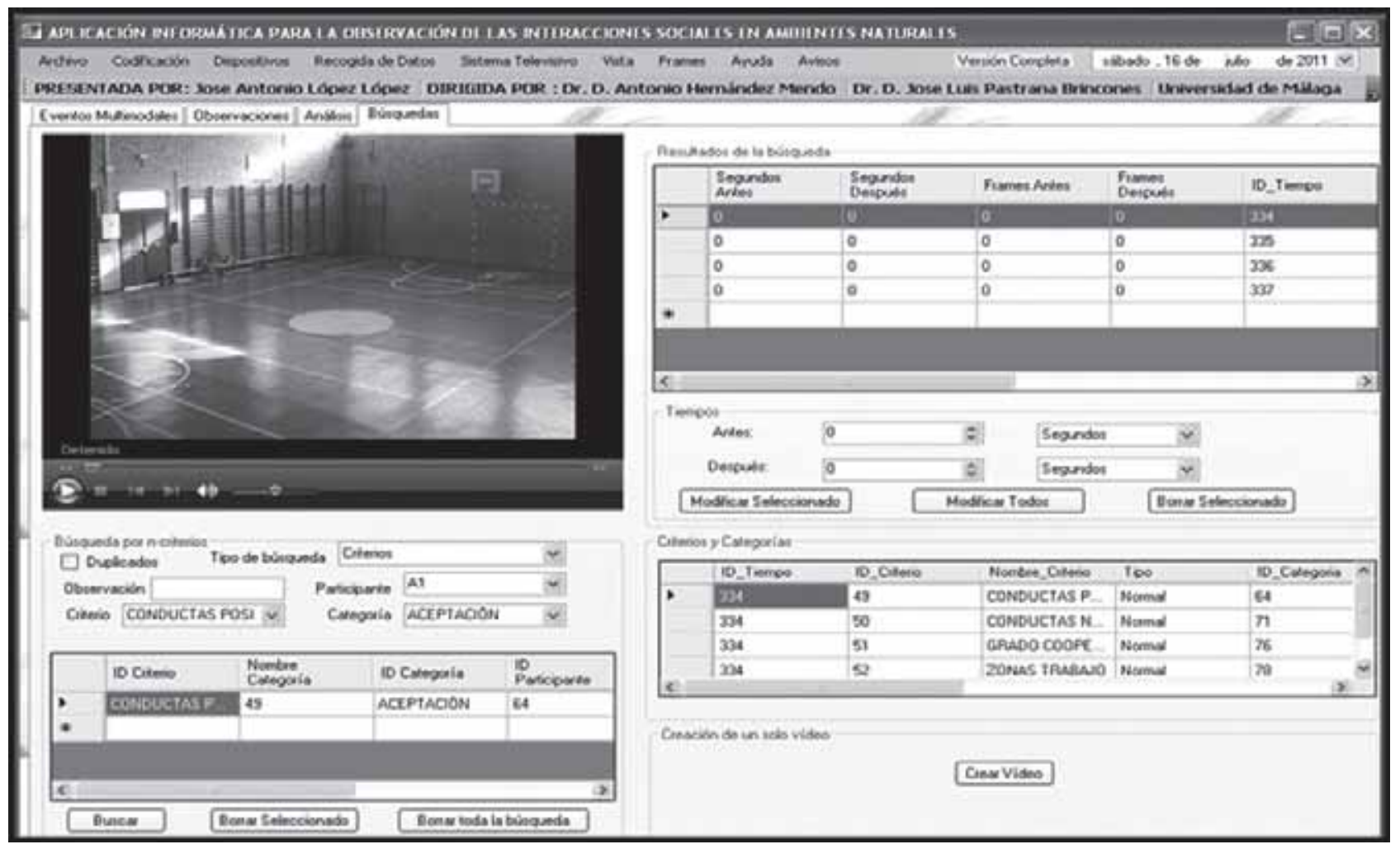

Finalmente, pinchando en el botón "Crear Vídeo", se puede crear un vídeo con la unión de los vídeos de todas las conductas encontradas en la búsqueda. Véase la Figura 28. 
Figura 28: Creación de Vídeo

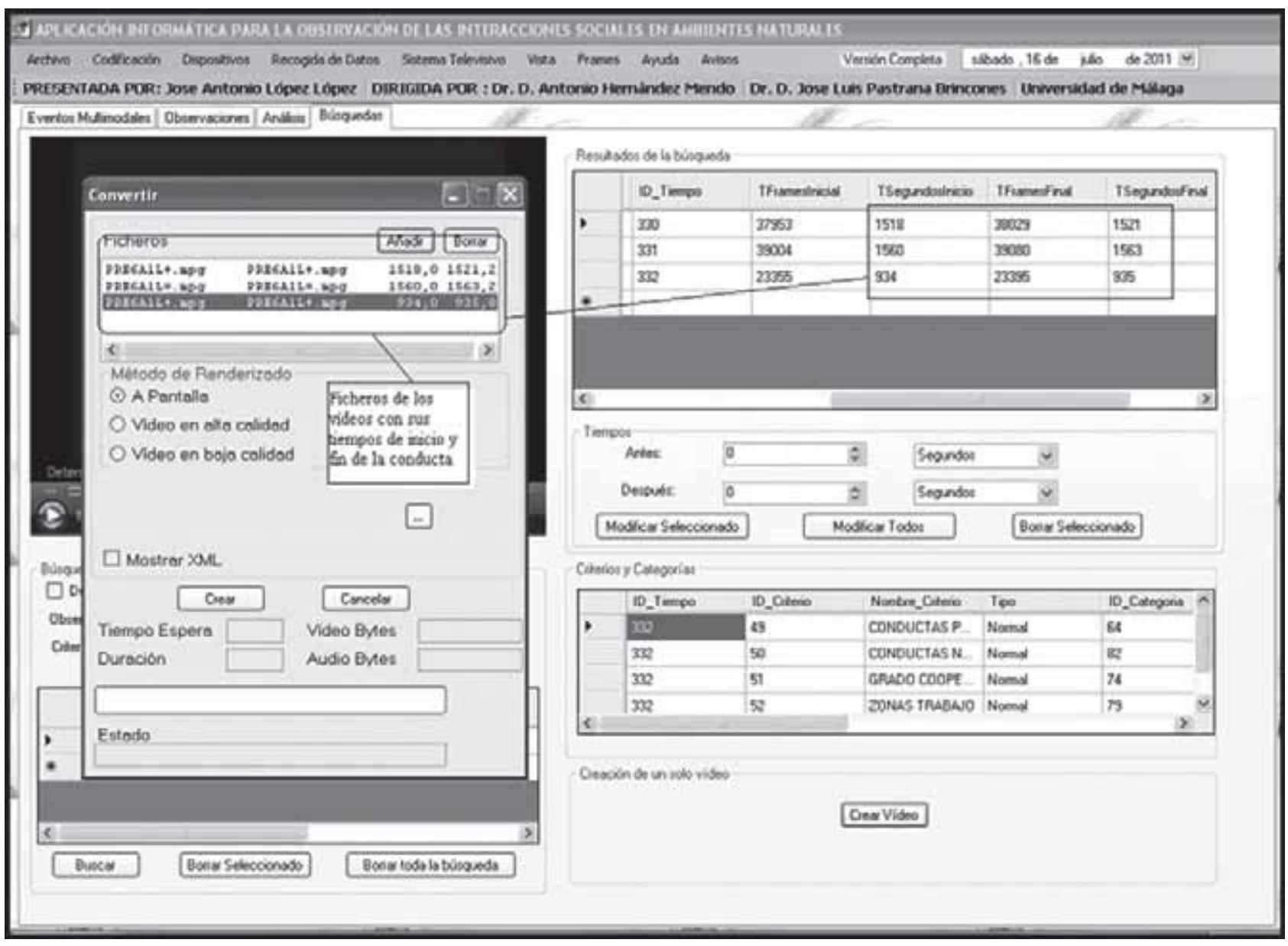

Como complemento a la descripción de el programa HOISAN, y cumplimentando uno de los objetivos -demostrar la funcionalidad del software- se ha realizado una comparativa del análisis de calidad de dato con HOISAN, SPSS y SDIS a partir de los registros obtenidos con el programa de observación MOTS (Castellano et al, 2008)

\section{MÉTODO}

\section{Participantes}

La estimación de la calidad del dato ha sido realizada utilizando la primera parte de un partido de fútbol 7 , correspondiente al campeonato escolar de Álava. En dicho encuentro, participaron escolares de categoría benjamín del C.D. Ariznabarra y el Deportivo Alavés. Cada sesión de observación ha supuesto el registro de más 2.100 códigos, agrupados en 350 codificaciones de secuencias de multieventos.

\section{Material}

El partido fue grabado con una videocámara JVC GZMG730 apoyada sobre un trípode y ubicada en una grada del campo de fútbol. También se utilizó un ordenador portátil con procesador Intel Core 2 Duo, 2.26 GHz, 4 GB de Ram y 320 GB de disco duro. Posteriormente, lo acontecido en el juego fue observado, codificado y registrado con el programa informático MOTS (Castellano, Perea, Alday y HernándezMendo, 2008). Para el análisis estadístico de la calidad del dato se ha exportado los registros a la aplicación HOISAN, SPSS v.19 y SDIS-GSEQ 5.0 con el que se han estimado los diferentes índices de asociación.

\section{Herramienta de observación}

La herramienta taxonómica está configurada por 7 criterios y 58 categorías repartidas en: Criterio 1 (14 niveles): inicio de la jugada; Criterio 2 (2 niveles): equipo con posesión del 
balón; Criterio 3 (8 niveles): zona de inicio de cada posesión individual; Criterio 4 (8 niveles): zona de finalización de cada posesión individual; Criterio 5 (12 niveles): jugador que lleva a cabo la jugada con balón; Criterio 6 (8 niveles): número de contactos del jugador con el balón; y, Criterio 7 (6 niveles): conducta con la que concluye la posesión individual. Los datos son del tipo multimodal.

\section{Procedimiento}

Tras la grabación del partido, se hizo necesaria la conversión del dispositivo digital en formato ${ }^{*}$.mod al formato ${ }^{*}$.mpeg, para poder ser visualizado con el programa MOTS. Con este programa se realizó el registro y codificación del flujo conductual por parte de dos observadores. Esto permitió estimar la concordancia interobservadores.
Figura 29. Interface del programa informático MOTS (Castellano et al, 2008)

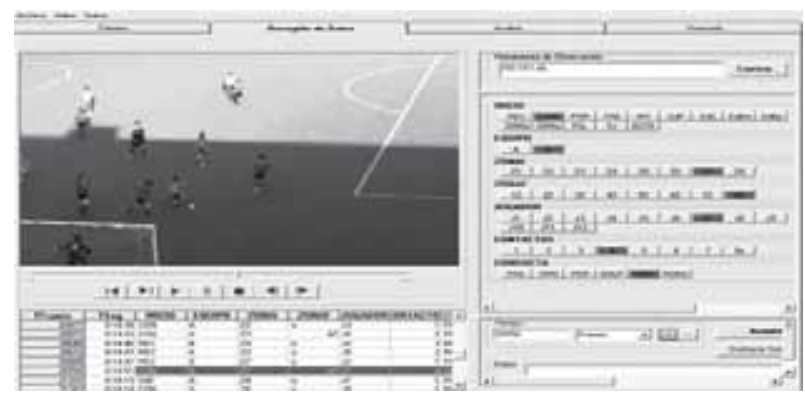

Tras el registro de los datos, se procedió al análisis de la calidad del dato a partir de los dos ficheros resultantes de la observación de la mitad del partido. Para ello, se emplearon los índices de asociación en la línea de otros estudios (Perea, Castellano, Hernández-Mendo, Álvarez y Pérez, 2005; Reina, Hernández-Mendo y Fernández-García, 2009). En esta vertiente, y a modo de ejemplo, hemos empleado el índice Kappa de Cohen. Se ha estimado dicho valor para cada uno de los criterios que configuran la herramienta de observación, así como para el total de la sesión. Para ellos se exportaron los registros a las diferentes aplicaciones. Tanto el SPSS como SDIS-GSEQ necesitan que la salida de datos del programa MOTS sean modificados para que puedan ser leídos, en tanto que el programa HOISAN incluye una opción para importar los datos directamente (figura 30) desde dicha aplicación.

Figura 30. Interface del programa HOISAN para importar los datos del MOTS.

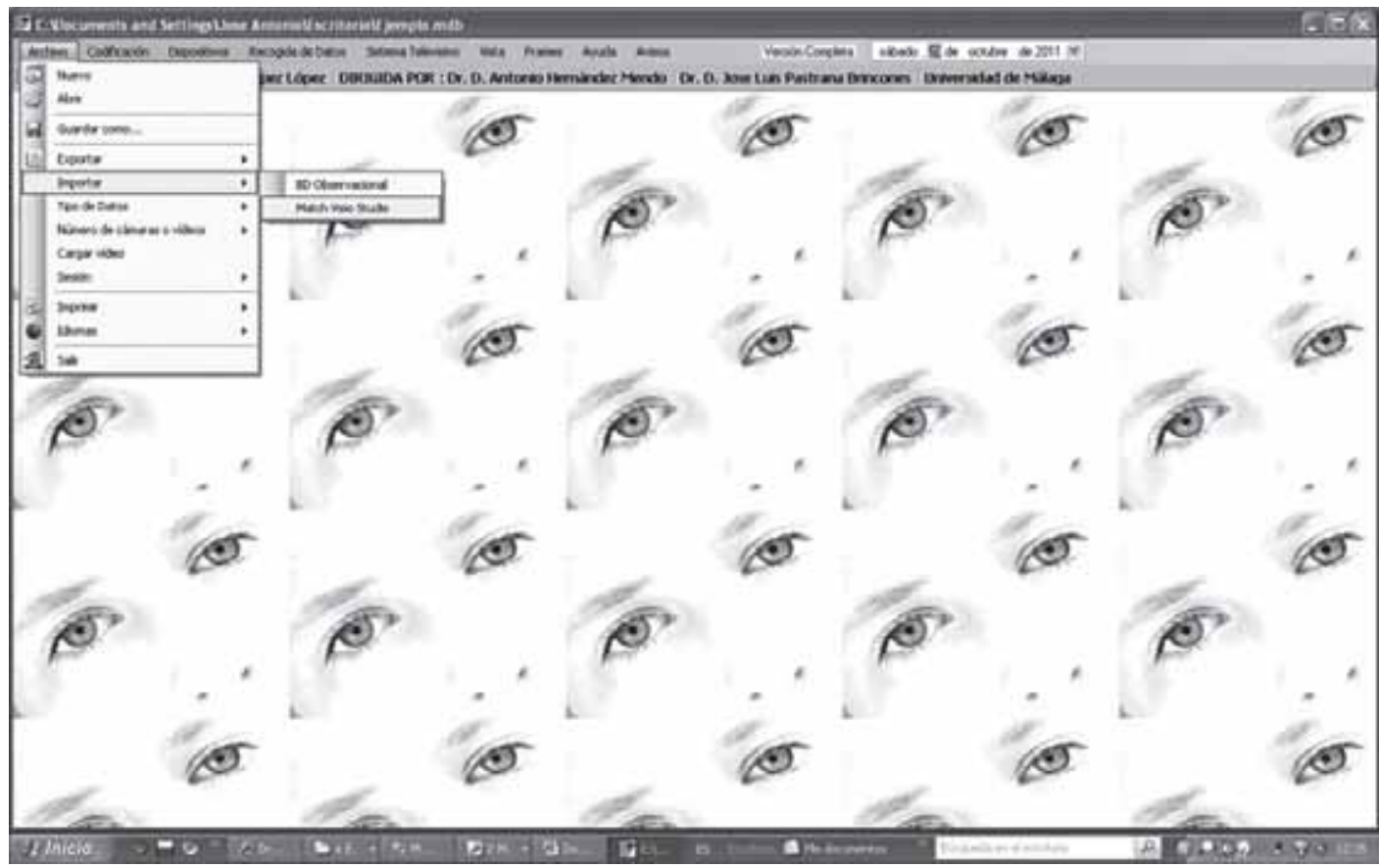


Una vez la herramienta taxonómica es reconocida por HOISAN (figura 31), permitiría continuar con la observación, codificación y registro de los datos (figura 32).

Figura 31. Interface del programa HOISAN con la herramienta de observación importada del MOTS.

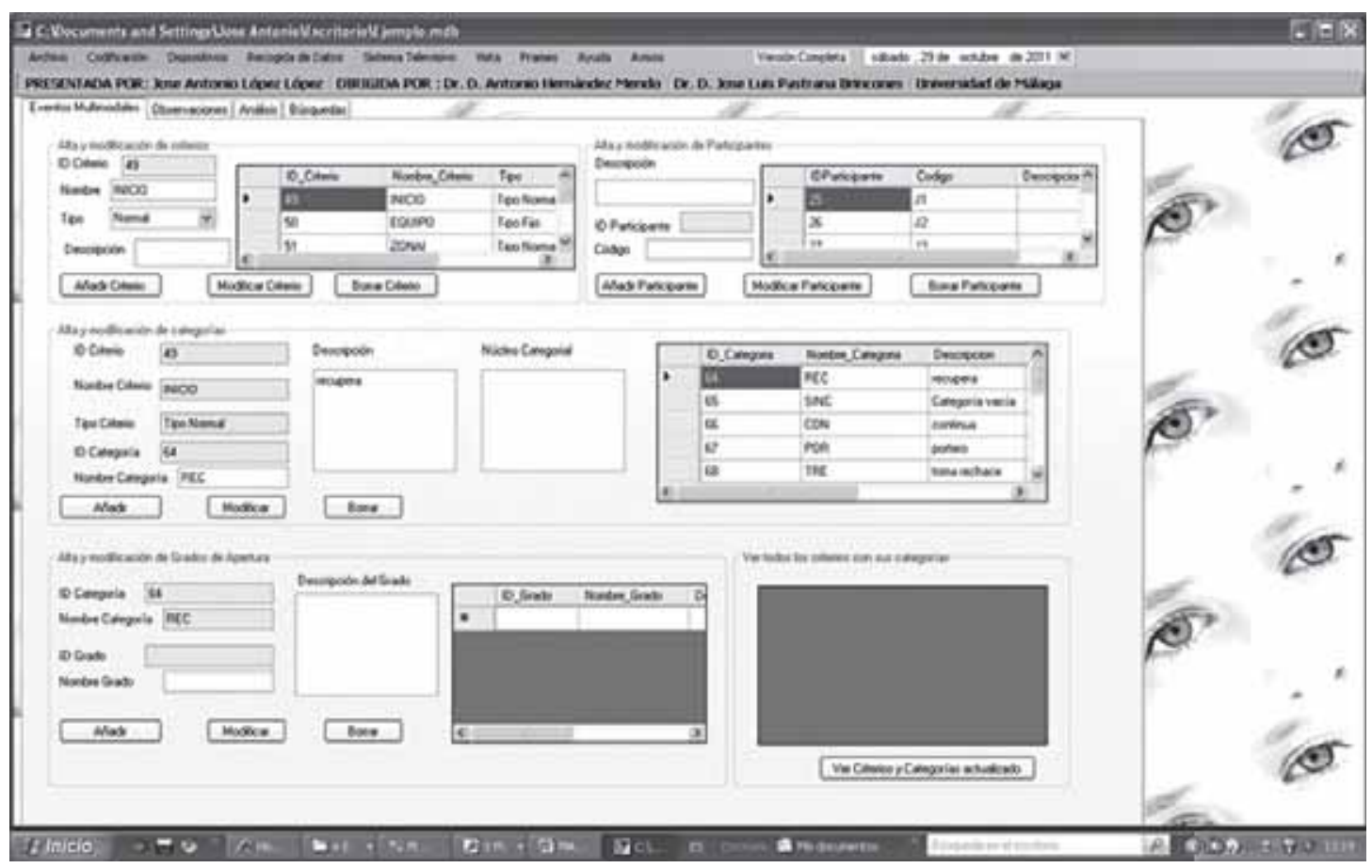

Figura 32. Interface del programa HOISAN donde se registra la codificación.

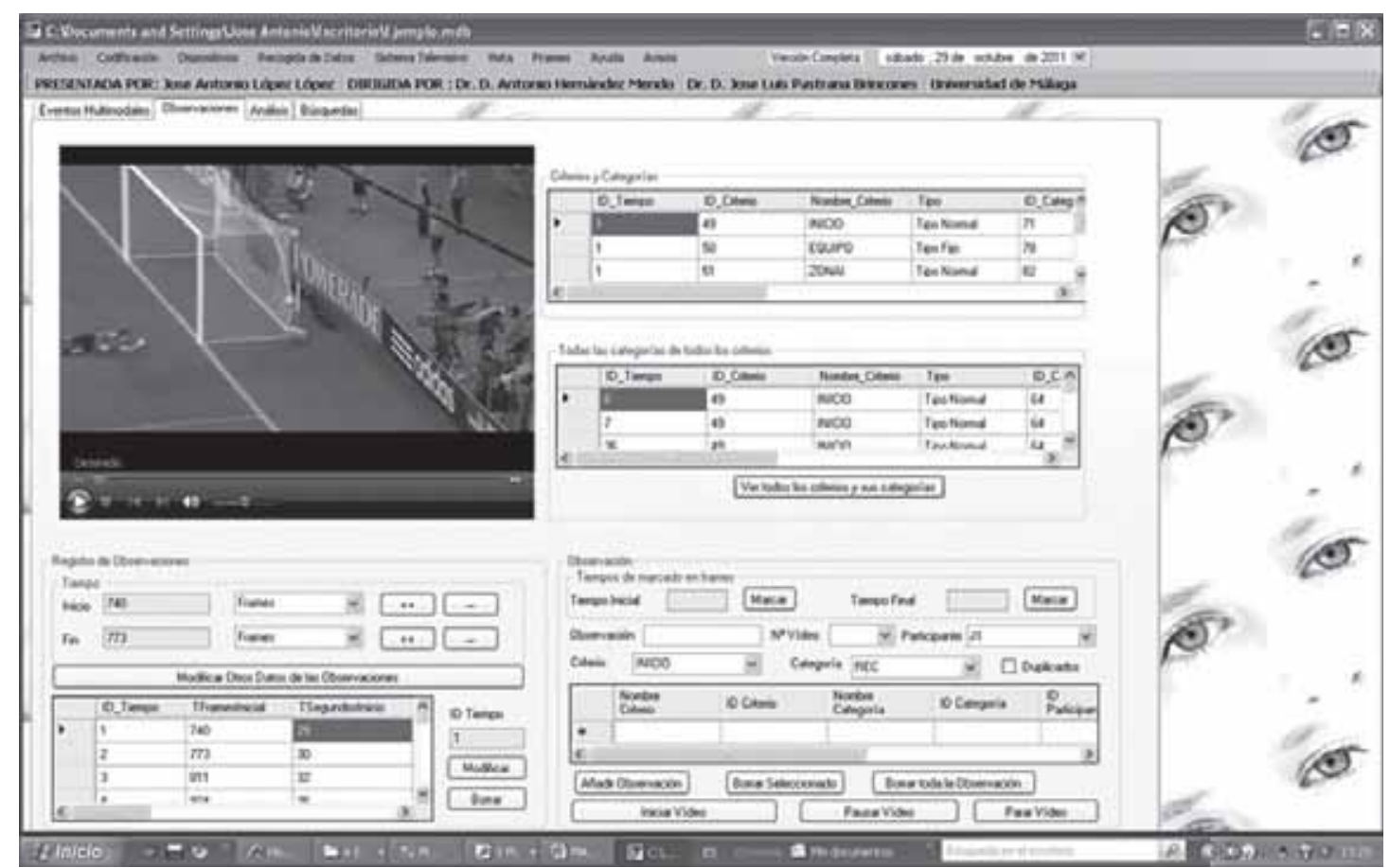


Finalmente, cuando la herramienta de observación y los archivos de registro que se obtienen del MOTS son importados por programa el HOISAN, se procede a estimar todo el repertorio de índices y correlaciones que permite la aplicación para todos los criterios, categorías y participantes (figura 33). Los valores del análisis aparecen en el epígrafe de resultados.

Figura 33. Interface del programa HOISAN donde se estiman los diferentes índices para valorar la calidad del dato.

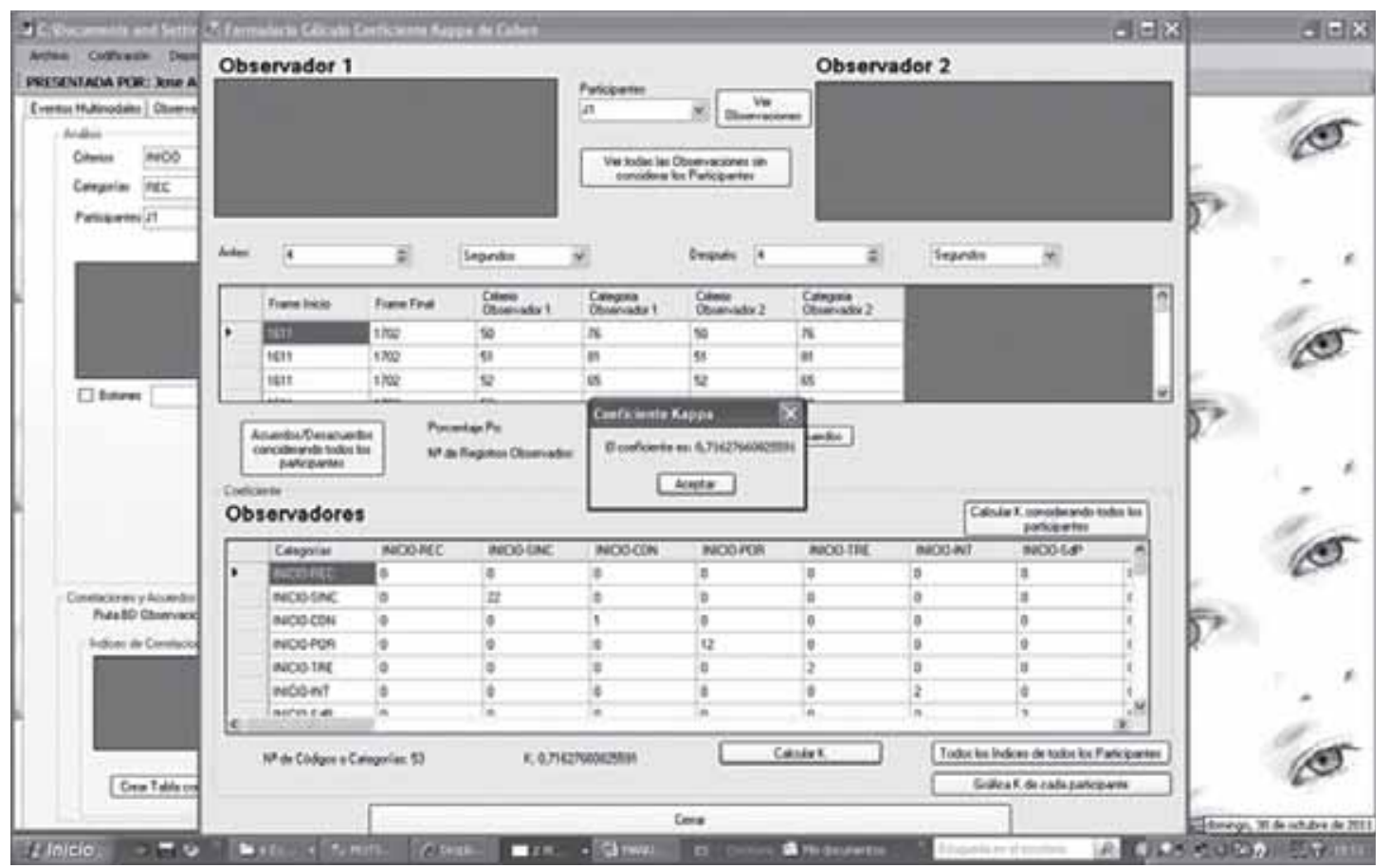

\section{Resultados}

En la tabla 13 se muestran los coeficientes de Kappa de Cohen para el total de la sesión estimados a partir de las tres aplicaciones (HOISAN, SPSS y SDIS-GSEQ). Cuando no se considera la variable tiempo de registro los valores estimados por las tres aplicaciones son similares $0.91,0.94$ y 0.90 , para el HOISAN, SPSS y SDIS-GSEQ respectivamente. 
Tabla 13. Salida de las aplicaciones HOISAN, SPSS y SDIS-GSEQ para el cálculo de la Kappa de Cohen

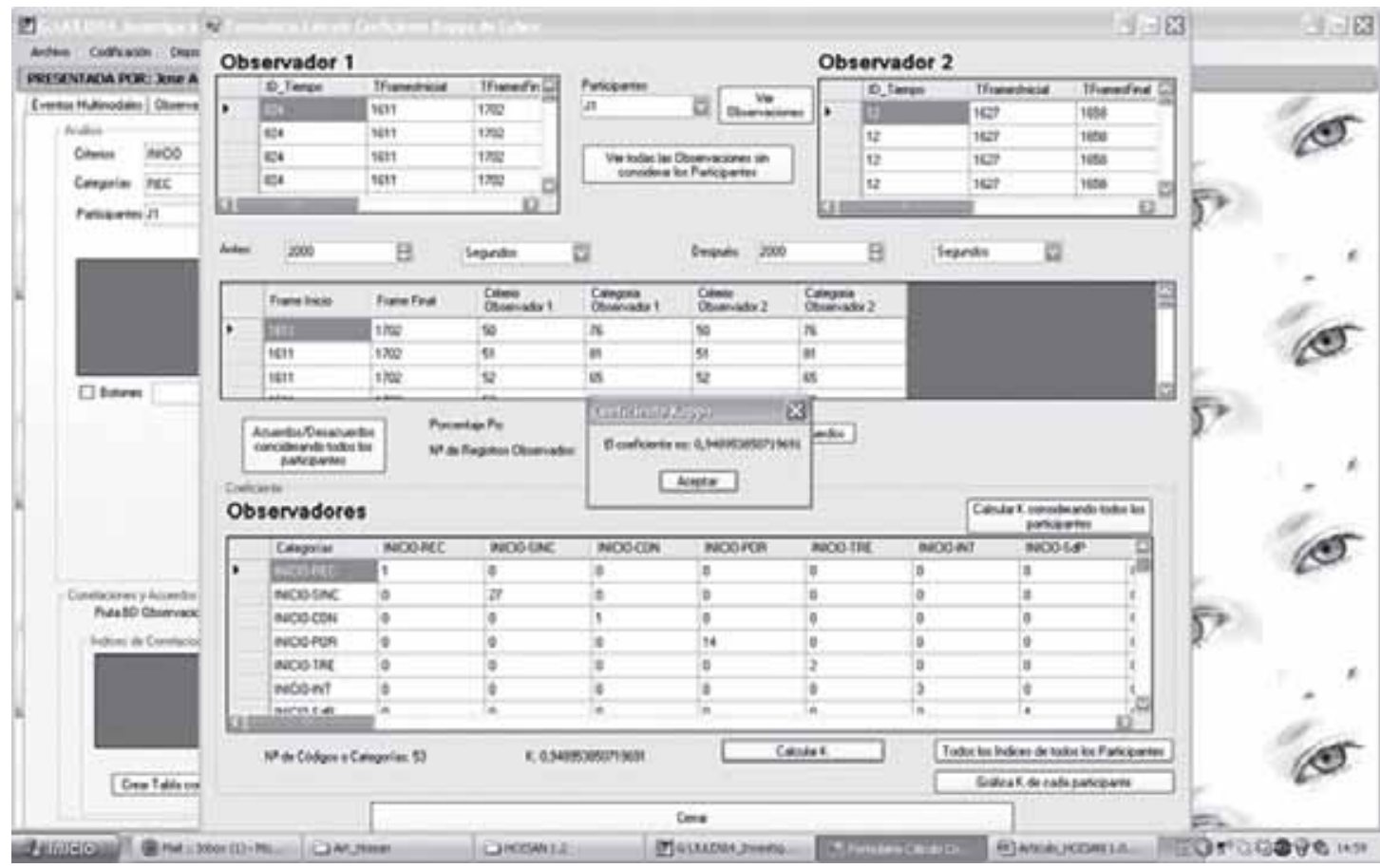

\section{Medidas simétricas}

Medida de acuerdo

Kappa $\quad$ Valor

Error típ. asint.

, 005

T aproximada ${ }^{\mathrm{b}}$ 190,838

Sig. aproximada

$\mathrm{N}$ de casos válidos

2034

a. Asumiendo la hipótesis alternativa.

b. Empleando el error típico asintótico basado en la hipótesis nula.

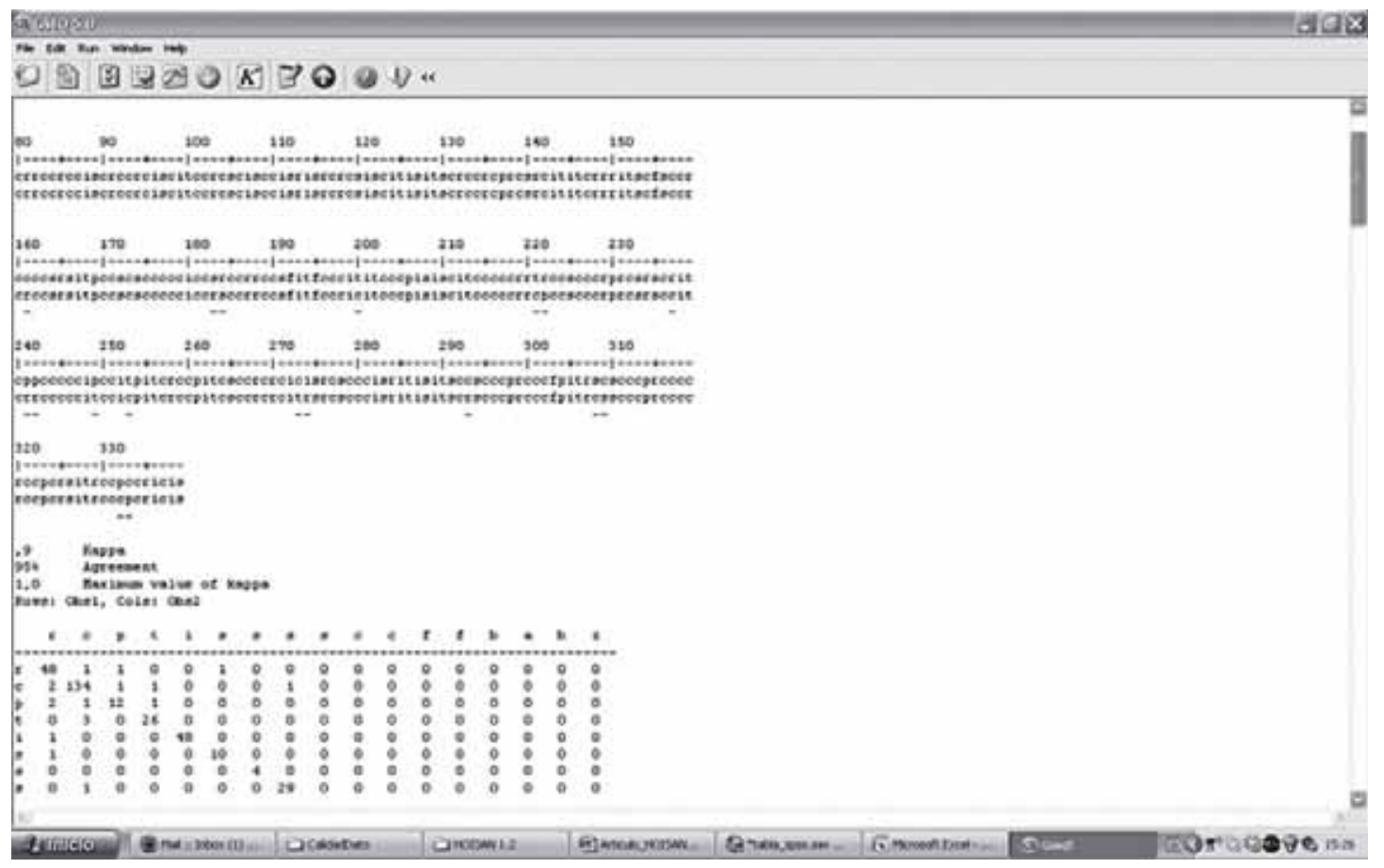


Los problemas detectados en el cálculo del índice Kappa en el SPSS es la exigencia de una tabla de contingencia cuadrada, es decir, el observador 1 y el 2 deben haber registrado el mismo número de categorías diferentes, de lo contrario la aplicación no puede mostrar un resultado, y esto, cuando los registros no son muy numerosos puede ocurrir. Por otro lado, en la aplicación SDIS-GSEQ, cuando la herramienta de observación está configurada por diferentes criterios el índice Kappa estimado para el conjunto de las categorías es la misma que para las categorías que se ubican en el primer criterio, con lo cual resulta imposible estimar Kappa para el conjunto de los criterios en el total de la sesión. Al contrario, la aplicación HOISAN, además del cálculo de Kappa, de otros índices, indicadores y/o coeficientes (como acuerdos, Pearson, Tau de Kendall, Spearman y Pi) puede calcularlo para cada uno de las observaciones, participantes o criterios. Finalmente, otras de las ventajas del HOISAN es permitir cierto gado de holgura cuando se compara la fiabilidad de dos registros, y en las primeras fases de la investigación, se ha considerado no contemplar el tiempo preciso de registro, ya que las duración de las conductas no interesa para resolver el problema de estudio. En la siguiente tabla 14 se recogen los valores de la Kappa y el coeficiente Pi estimados con HOISAN.

Tabla 14. Valores de la Kappa de Cohen y el coeficiente Pi para los diferentes intervalos de holgura.

\begin{tabular}{lcc}
\hline Intervalo de holgura (segundos) & Kappa de Cohen & $\mathrm{Pi}$ \\
\hline 0 & 0.23 & 0.16 \\
\pm 2 & 0.41 & 0.54 \\
\pm 4 & 0.64 & 0.73 \\
\pm 6 & 0.70 & 0.74 \\
\pm 8 & 0.72 & 0.75 \\
\pm 10 & 0.75 & 0.79 \\
$2000^{*}$ & 0.91 & 0.95 \\
${ }^{*}$ Cuando no se tiene en cuenta el tiempo. &
\end{tabular}

El repertorio de posibilidades analíticas para estimar la calidad del dato aportadas por el HOISAN es muy amplio y de fácil aplicación. Basta un click de ratón para obtener todo un espectro de indicadores sobre la fiabilidad de los registros, de esta forma evitamos tener que exportar los datos a otras aplicaciones informáticas para la estimación de los mismos.

\section{Conclusiones}

A modo de síntesis final presentamos las ventajas más destacadas que aporta la el software presentado, HOISAN, en el intento de dar respuesta a las necesidades de investigadores y cuerpos técnicos a múltiples niveles, como por ejemplo:

Permite la construcción de herramientas observaciones, bien como sistema de categorías E/ME o como sistema de formatos de campo, utilizando todos los tipos de datos (Bakeman y Quera, 1995), garantizando criterios científicos de fiabilidad y validez, pudiendo ser aplicados a los diversos contextos sociales donde se produce la interacción.

Su flexibilidad, tanto para la realización de la observación (ya sea de grabaciones o de una o varias cámaras en tiempo real) como en el tipo de datos a utilizar (Secuencias de Estados, Eventos, Mixtas, Intervalos de Tiempo y Eventos Multimodales). HOISAN puede utilizar hasta 20 vídeos simultáneamente (grabados previamente) o hasta 20 cámaras para realizar observación en vivo, esta característica permite que herramientas como los formatos de campo o las secuencias de eventos multimodales adquieran su verdadero sentido y potencialidad.

Gran variedad de análisis, con el uso de parámetros primarios y medidas derivadas o secundarias, cálculo de índices de correlación, coeficientes, fiabilidades y análisis de la producción verbal.

La implementación se ha desarrollado mediante el lenguaje C\# y la plataforma .NET. La gran ventaja de este tipo de programación es la sencillez, se usa un lenguaje moderno y orientado a objetos. La plataforma tiene la capacidad para soportar aplicaciones grandes y complejas. Intercambio rápido de los datos generados por la aplicación con otros programas como son (Excel, SPSS, Word, Atlas.Ti, Sdis-Gseq, MOTS, Observer y Theme). También exporta a PDF.

Permite seguir de forma nítida, clara y sencilla el proceso de estructuración categorial (definición de los núcleos categoriales, grados de apertura y participantes) así como las pautas previas de los diseños observacionales (Anguera, Blanco Villaseñor, Hernández Mendo, y Losada, 2011).

Tiene implementada una opción para el tratamiento o exportación de datos cualitativos, ya que la MO comparte características de la Metodología Cualitativa (Anguera, 2004). HOISAN realiza un primer análisis de los datos almacenados en el campo observaciones y los exporta directamente al programa de análisis de datos cualitativos Atlas.ti en formato ".xls". Utilizando este formato de exportación y la opción de Atlas.ti Import Survey Data (Importar Datos de Cuestionario) en el menú Assign (Asignar) en la barra de herramientas o en el menú Documents (Documentos).

Actualmente se está trabajando en realizar mejoras de los algoritmos creados e implementaciones futuras de nuevos algoritmos para el cálculo de nuevos índices de correlación y el cálculo de coordenadas polares (Hernández Mendo y Anguera, 1998; Castellano y Hernández Mendo, 2003). El programa es de libre distribución y se puede descargar gratuitamente de la plataforma www.menpas.com http://www.menpas.com/ Eval_Individual/Observacion/Hoisan.aspx 


\section{Referencias}

Anguera, M. T. (1988). Observación en la escuela. Barcelona: Graó.

Anguera, M. T. (1990). La metodología observacional. En J. Arnau, M. T. Anguera y J. Gómez (Eds), Metodología de la investigación en Ciencias del Comportamiento (pp. 7 -122). Murcia: Universidad de Murcia. Secretariado de Publicaciones.

Anguera, M. T. (1995). Metodología cualitativa. En M. T. Anguera, J. Arnau, M. Ato, R. Martínez, J. Pascual y G. Vallejo, Métodos de investigación en Psicología (pp. 513-522). Madrid: Síntesis.

Anguera, M. T. (2004). Posición de la metodología observacional en el debate entre las opciones metodológicas cualitativa y cuantitativa. ¿Enfrentamiento, complementariedad, integración? Psicologia em Revista (Belo Horizonte, Brasil), 10(15), 13-27.

Anguera, M. T. (2004). Posición de la metodología observacional en el debate entre las opciones metodológicas cualitativa y cuantitativa. ¿Enfrentamiento, complementariedad, integración? Psicologia em Revista (Belo Horizonte, Brasil), 10(15), 13-27.

Anguera, M. T.; Blanco A. y Losada, J. L. y Hernández Mendo, A. (2000). La metodología observacional en el deporte: conceptos básicos. Lecturas: EF y Deportes. Revista Digital, 24, agosto 2000. http://www.efdeportes.com/efd24b/obs.htm [Consulta: 10 de octubre de 2000].

Anguera, M. T., Blanco Villaseñor, A., Hernández Mendo, A., y Losada, J. L. (2011). Diseños observacionales: ajuste y aplicación en psicología del deporte. Cuadernos de Psicología del Deporte, 11(2),63-76.

Bakeman, R. y Gottman, J. M. (1987). Applying observational methods: a systematic view. In J.D. Osofsky, Handbook of infant development. New York: Wiley.

Bakeman, R. y Quera, V. (1995). Amalyzing interaction: Sequential analysis using SDIS and GSEQ. New York: Cambridge University Press (Traducción española Análisis de la interacción. Análisis Secuencial con SDISGSEQ. Madrid: Ra-Ma.).

Bickman, L. y Henchy, T. (Eds.) (1972). Beyond the laboratory : Field research in social psychology. New York : McGraw-Hill.

Castellano, J. y Hernández Mendo, A. (2003). El análisis de coordenadas polares para la estimación de relaciones en la interacción motriz en fútbol. Psicothema, 15(4), 569-574.

Castellano, J., Perea, A., Alday, L., y Hernández Mendo, A. (2008). Measuring and observation tool in sports. Behavior Research Methods, 40(3), 898-905.

Cohen, J. (1968). Weighted kappa: Nominal scale agreement with provision for scaled disagreement of partial credit. Psychological Bulletin, 70(4), 213-220

Cook, T. D. y Reichardt, Ch. S. (1986). Métodos cualitativos y cuantitativos en investigación evaluativa. Madrid : Morata.

Hernández Mendo, A, Anguera, M. T. y Bermúdez-Rivera, M. A. (2000).
Software for Recording Observational Files. Behavior Research Methods Computers y Instruments, 32(3), 436-445.

Hernández Mendo, A. (1996). Observación y análisis de patrones de juego en deportes sociomotores. Santiago de Compostela: Servicio de Publicaciones e Intercambio Científico.

Hernández Mendo, A. y Anguera, M. T. (1998). Análisis de coordenadas polares en el estudio de las diferencias individuales de la acción de juego. En M.P. Sánchez López y M.A. Quiroga, Perspectivas actuales en la investigación psicológica de las diferencias individuales (pp.85-88). Madrid: Ed. Centro de Estudios Ramón Areces.

Hernández Mendo, A., Bermúdez Rivera, M. A., Anguera, M. T., y Losada, J. L. (2000). CODEX: Un programa informático para codificación de registros observacionales. Lecturas: Educación Física y Deportes, Revista Digital, 5, 18. Available at www.sportquest.com/ revista/efd18/codex. htm.

Hernández Mendo, A., Ramos, R., Peralbo, M., y Risso, A. (1993). Un programa para el análisis observacional: Transcriptor v1.1, aplicación en psicología del deporte. Revista de Entrenamiento Deportivo, 7, 18-25.

Lewin, K. (1948). Resolving social conflicts: Selected papers on group dynamics. NewYork: Harper y Row.

Magnusson, M. S. (1988). Le temps et les patters syntaxiques du comportament humain: modele, méthode et le programme THEME. Revue des Conditions de Travail, 19-20, 284-314.

McGuire, W. J. (1967). Some empendings reorientations in social psychology. Journal of Experimental Social Psychology, 3, 124-139.

McGuire, W. J. (1973). The ying and yang of progress in social psychology : Seven koan. Journal of Personality and Social Psychology, 26, 446-456.

Noldus, L. P. J. J. (1991). The Observer: A software system for collection and analysis of observational data. Behavior Research Methods, Instruments, and Computers, 23, 415-429.

Riba, C. (1991). El método observacional. Decisiones básicas y objetivos. En M.T. Anguera (Ed.), Metodología observacional en la investigación psicológica, Vol. I. (pp. 29-114). Barcelona: P.P.U.

Ring, K. (1967). Experimental Social Psychology: Some sober Questions about some frovolus values. Journal of Experimental Social Psychology, 3(2), 113-123.

Sackett, G. P. (1978) Observing Behavior (Vol. 2): Data collection and analysis methods. Baltimore: University of Park Press.

Silverman, I. (1977). Why social psychology fails. Canadian Psychological Review, 18, 353-358.

Suen, H. K., y Ary, D. (1989). Analyzing quantitative behavioral observation data. Hillsdale, NJ: Erlbaum.

Taylor, S. J. y Bogdan, R. (1992). Introducción a los métodos cualitativos de investigación. Barcelona. Paidós. 